\title{
Companionship during facility-based childbirth: results from a mixed-methods study with recently delivered women and providers in Kenya
}

Patience Afulani ${ }^{1,2^{*}}$ (D) Caroline Kusi ${ }^{2}$, Leah Kirumbi ${ }^{3}$ and Dilys Walker ${ }^{1,2}$

\begin{abstract}
Background: Research suggests that birth companionship, and in particular, continuous support during labor and delivery, can improve women's childbirth experience and birth outcomes. Yet, little is known about the extent to which birth companionship is practiced, as well as women and providers' perceptions of it in low-resource settings. This study aimed to assess the prevalence and determinants of birth companionship, and women and providers' perceptions of it in health facilities in a rural County in Western Kenya.

Methods: We used quantitative and qualitative data from 3 sources: surveys with 877 women, 8 focus group discussions with 58 women, and in-depth interviews with 49 maternity providers in the County. Eligible women were 15 to 49 years old and delivered in the 9 weeks preceding the study.

Results: About $88 \%$ of women were accompanied by someone from their social network to the health facility during their childbirth, with $29 \%$ accompanied by a male partner. Sixty-seven percent were allowed continuous support during labor, but only $29 \%$ were allowed continuous support during delivery. Eighteen percent did not desire companionship during labor and 63\% did not desire it during delivery. Literate, wealthy, and employed women, as well as women who delivered in health centers and did not experience birth complications, were more likely to be allowed continuous support during labor. Most women desired a companion during labor to attend to their needs. Reasons for not desiring companions included embarrassment and fear of gossip and abuse. Most providers recommended birth companionship, but stated that it is often not possible due to privacy concerns and other reasons mainly related to distrust of companions. Providers perceive companions' roles more in terms of assisting them with non-clinical tasks than providing emotional support to women.

Conclusion: Although many women desire birth companionship, their desires differ across the labor and delivery continuum, with most desiring companionship during labor but not at the time of delivery. Most, however, don't get continuous support during labor and delivery. Interventions with women, companions, and providers, as well as structural and health system interventions, are needed to promote continuous support during labor and delivery.
\end{abstract}

Keywords: Continuous support during labor and delivery, Birth companionship, Woman-centered care, Respectful maternity care, Kenya

\footnotetext{
* Correspondence: patience.afulani@ucsf.edu

${ }^{1}$ Obstetrics, Gynecology and Reproductive Sciences, UCSF, San Francisco,

USA

${ }^{2}$ Institute for Global Health Sciences, UCSF, San Francisco, USA

Full list of author information is available at the end of the article
}

(c) The Author(s). 2018 Open Access This article is distributed under the terms of the Creative Commons Attribution 4.0 International License (http://creativecommons.org/licenses/by/4.0/), which permits unrestricted use, distribution, and reproduction in any medium, provided you give appropriate credit to the original author(s) and the source, provide a link to the Creative Commons license, and indicate if changes were made. The Creative Commons Public Domain Dedication waiver (http://creativecommons.org/publicdomain/zero/1.0/) applies to the data made available in this article, unless otherwise stated. 


\section{Background}

Over the past decade, the promotion of facility based delivery has been at the forefront of international efforts to reduce maternal mortality. Between 2003 and 2013, the percentage of women who delivered in health facilities increased in every region of the world [1]. However, retention, equity, dignity, and quality of care along the continuum of maternal health care remain a challenge [2-4]. Women's experiences during childbirth are a powerful determinant of use of maternal healthcare services [5]. The World Health Organization (WHO) Quality of Care Framework for maternal and newborn health outlines access to emotional and social support of women's choice as core to the experience of care and to achieving positive person-centered health outcomes [6]. Thus, WHO recommends that every woman is offered the option to experience labor and childbirth with a companion of her choice [7].

Different terms have been used for this recommendation, including companion of choice at birth, labor companion, birth companion, emotional support during birth, social support during labor and delivery, supportive companionship, and continuous support for women during childbirth [8-13]. But the evidence supporting the recommendation emphasizes continuous support during childbirth $[9,10]$. The definition of continuous support during childbirth differs across studies and settings and can start at any time between conception to postpartum period. Generally, however, it involves the continuous presence of a companion during labor and delivery $[9,10]$. In this paper, we use the terms birth companionship to refer to the provision of any type of support by a lay companion at any time during the intrapartum period, and continuous support during labor and delivery (CSLD) to refer to the continuous presence of the companion during labor and delivery. CSLD is thus a subset of birth companionship since not all birth companionship is continuous.

Common elements of support provided by birth companions include, emotional support (e.g. continuous presence and giving reassurance and praise), informational support (e.g. giving information about labor progress and advice regarding coping techniques), comfort measures (e.g. comforting touch, massage, warm baths/ showers, encouraging mobility, and promoting adequate fluid intake and output), advocacy (e.g. helping women articulate their wishes to providers), and instrumental support (providing assistance with needs) [9, 10, 14]. Support can be provided by different types of companions, including trained support persons such as doulas (non-health care professionals trained to provide emotional and physical support to women before, during and after childbirth) or by companions chosen from a woman's social network $[9,15]$.
Birth companionship is associated with increased satisfaction with healthcare services $[9,16]$. Some studies also suggest that women who have birth companions are less likely to experience mistreatment during childbirth $[17,18]$. In addition, CSLD has been shown to impact pregnancy outcomes. A recent Cochrane review of CSLD found that women who received continuous labor support were more likely to have shorter labor and spontaneous vaginal deliveries (i.e. give birth vaginally without the use of instruments or to have a cesarean section). In addition, they were less likely to use pain medication while giving birth and their babies were less likely to have low five-minute Apgar scores [9]. The effect of CSLD differed, however, depending on the type of companion and the setting. They found that CSLD was most effective at reducing caesarean birth when the companion was present in a doula role, as well as in settings where epidural analgesia was not routinely available. The effect of CSLD on birth outcomes was also stronger in settings where women were not permitted to have companions during labor. The authors concluded that continuous support from a person who solely provides support, is not a member of the woman's own network, is experienced in providing labor support, and has a modest amount of training (such as a doula), appears to have the greatest impact on outcomes. Nonetheless, compared to having no companion, support from a chosen family member or friend appeared to increase women's satisfaction with their childbirth experience [9]. The authors noted that none of the studies included in their review were from low-income settings and, therefore, recommended that future studies explore how continuous support can be best provided in different contexts, particularly in low-income settings.

Birth companionship is increasingly being included in the maternal health guidelines of many countries, including in low- and middle-income countries in Sub-Saharan Africa (SSA). For example, the National Guidelines for Quality Obstetrics and Perinatal Care in Kenya recommends that during the first stage of labor, providers should encourage women to have their chosen companions with them and make arrangements to accommodate birth companions or male partners; and in the second stage of labor providers should "allow and encourage her birth companion/male partner to be present" [19]. Yet little is known about the extent to which this is actually practiced in Kenya, as well as in most of SSA. The research is equally limited on women's perceptions and preferences for birth companions, and on providers' perceptions of birth companions in these settings [11-13, 20,21]. No study to our knowledge has comprehensively examined birth companionship in Kenya. Little is therefore known about the prevalence of birth companionship including CSLD, the types of companions used, women's preferences for 
companionship, and the factors associated with these in Kenya. In contexts where women have little autonomy and their health seeking behavior is strongly influenced by sociocultural norms; and providers have greater autonomy, but operate with very limited resources, it is important to understand women's preferences for companionship and providers perceptions about providing it, to guide efforts towards promoting birth companionship. The current study aims to fill this gap in evidence.

Furthermore, while most of the works on birth companionship have examined it as a single unit, we chose to make some distinctions based on our field experiences. The first distinction is between having a companion present and having continuous support. This is motivated by the fact that, while a woman might have a companion present, the support provided may not be continuous for various reasons. The second distinction is between labor support, delivery support, and postdelivery support. This distinction is to enable us to understand if women's preferences for support differ at these different stages of the childbirth process. In addition, these distinctions are important because, when support is not continuous and is only provided at certain time points (like during labor but not delivery), the potential benefits may differ from those stated in CSLD literature. Our objectives, therefore, were to: (1) Assess the prevalence of birth companionship and CSLD in health facilities in a rural county in western Kenya; (2) Assess women's desire for birth companionship; (3) Identify types of birth companions that women prefer; and (4) Examine factors associated with receiving CSLD, desire for companionship, and preference for male companions. In addition, we use qualitative data to examine: (1) women's perceptions of birth companionship and their preferred companions; and (2) providers' perceptions of birth companionship and barriers to ensuring CSLD for women.

\section{Methods}

We employed mixed methods for this study. Mixed methods combine elements of qualitative and quantitative approaches to provide breadth and depth in our understanding of phenomenon [22]. Quantitative methods allow objective measurement of a particular reality, with a large enough sample to increase generalizability [23]. Quantitative methods are however limited in explaining the "why" of phenomena and how personal viewpoints, context and meaning produce multiple realities [23]. Qualitative methods help to address this weakness, but have limited generalizability because of practical limitations of sample size. Mixed methods were therefore appropriate to harness the strengths and counterbalance the weaknesses of quantitative and qualitative methods to provide a holistic understanding of birth companionship [23].
The quantitative methods provide data for measuring prevalence and examining statistical associations and the qualitative data help to explain the quantitative findings and provide rich descriptions of views and beliefs about birth companionship. In addition, we collected data from recently delivered women and maternity providers to obtain the complementary perspectives of both the recipients and providers of care.

\section{Setting}

Data for this study were part of a larger study examining community perceptions of quality of maternity care in a rural county in western Kenya. The County has a population of about one million and an estimated 40,000 annual births [24]. Approximately $43 \%$ of the population live below the poverty line and only about $3 \%$ of women of reproductive age have more than a secondary education. About 24\% of women of childbearing age are 15-19 years [25]. The total fertility rate of the county is 5.3 compared to the national average of 3.9. The County is one of 15 counties in Kenya that account for over $60 \%$ of maternal deaths in the country-with an estimated maternal mortality ratio of 673 deaths per 100,000 live births compared to the national average of 495 [26]. The number of nurses, clinical officers, and doctors, per 100,000 people in the county is 32,19 , and 4 respectively [27].

About $53 \%$ of births in the County occur in health facilities, compared to the national average of $61 \%$ [25]. Several factors account for low use of facility deliveries, including low perceived need of facility deliveries, poor physical and financial access to health services, and low perceived quality of care [28-30]. Additionally, sociocultural factors such as norms and values related to childbirth (e.g. beliefs about the causes of pregnancy complications) and women's autonomy (e.g. women not being final decision makers on place of birth) interact with socioeconomic factors such as wealth and education to determine whether or not women seek health care during pregnancy or delivery [5, 31-33]. Thus, the low status of most childbearing women in the County (young, uneducated, poor, and unemployed) is likely a key determinant of the low use of services in the County. These factors also affect how women are treated in the health facility, which affects future health seeking behavior. Given evidence that birth companionship improves women's birth experiences $[5,9,17]$, understanding and respecting women's desires and preferences for birth companions could positively impact health seeking behavior during childbirth in the County.

\section{Data sources}

We used 3 data sources: surveys and focus group discussions with recently delivered women and in-depth interviews with providers. Eligible women were 15-49 years and 
delivered in the 9 weeks preceding the study. Eligible providers worked in a maternity unit in the county. Ethical approval for the study was provided by the authors' institutions. All participants provided written informed consent after receiving information about the study and received $200 \mathrm{KES}(\sim \$ 2)$ for their participation.

\section{Surveys with women}

Survey data were collected between August and September 2016 with eligible women. A multistage sampling approach was used to select women. First, the County was divided into eight strata based on its 8 sub-counties. Next, ten health units were randomly selected from each strata. Eligible women were then identified and recruited from the selected health units. The sampling procedures are documented elsewhere [34]. Twelve data collectors were trained to conduct the interviews-in English, Swahili, and Luo in private spaces in health facilities or in respondents' homes. Data were collected using the REDCap electronic data capture tools hosted at the University of California-San Francisco [35]. Approximately one thousand women were interviewed, with a response rate above $98 \%$. We use data from women who delivered in a health facility (894) and had no missing data on relevant variables for this analysis ( $N=857-877$ for different outcomes).

\section{Focus group discussions (FGDs) with women}

Between October and November 2016, we conducted eight FGDs-one in each sub-county-with 58 eligible mothers. We chose a focus group design to obtain qualitative data from women because our field experience in the region suggested women were more willing to discuss their experiences in groups with their peers than with an individual interviewer not familiar to them. The FGD procedures are documented elsewhere [36]. We recruited respondents from one randomly selected health unit in each sub-county that was not included in the survey. Women who met the eligibility criteria were purposively selected from the selected health unit. Each focus group consisted of six to ten women and lasted about $90 \mathrm{~min}$. Two female research assistants with college degrees were trained by the first author to moderate each group discussion; one led the discussion using a discussion guide, and the other took notes and managed audio recording. Women were asked, among other things, if they were allowed to have a companion present during labor and delivery and how they felt when they were allowed or disallowed a companion. They were also asked if they desired birth companionship and why they desired or did not desire it. The discussions were conducted in Swahili or Luo, in private spaces in the community or health facility. Discussions were audio recorded, and simultaneously translated and transcribed.

\section{In-depth interviews with providers}

We conducted 49 interviews with maternity providers from 18 facilities across all the 8 sub-counties, which were selected for an intrapartum quality improvement project based on their relatively higher volume of deliveries. With permission from the county and facility heads to conduct the study, the research assistants approached maternity providers in selected facilities, briefed them about the study, and invited them to participate in the interviews. The interviewers used an interview guide with both closed and open-ended questions. Providers were asked, among other things, if women were allowed CSLD, their perceptions of CSLD, and barriers to providing it in their facilities. Interviews were conducted in English, Swahili or Luo-in private spaces in each health facility-and lasted about an hour. The structured responses were directly entered into the REDCap application during the interview. Interviews were also audio-recorded and transcribed (with simultaneous translation where necessary).

\section{Measures for quantitative analysis Dependent (outcome) variables}

Our dependent variables are from several survey questions on birth companionship during: (1) labor, (2) delivery, and (3) post-delivery. These include questions on whether they were accompanied by a companion to the facility, their relationship to the companion, whether they were allowed continuous support during labor and during delivery, whether they desired companionship during labor, delivery, and after delivery, and their preferred type of companion. The variables are described in Table 1 with exact wording of questions in Additional file 1.

\section{Independent variables}

These include various socio-demographic, facility, and provider related factors that might be associated with the dependent variables. These were selected based on existing literature and theoretical reasoning. They include age, marital status, parity, education, literacy, employment, household wealth, tribe, religion, prior facility delivery, antenatal attendance, pregnancy complications, labor and delivery complications, type of delivery facility, type and sex of delivery providers, and women's perceptions of crowding in the facility. In multivariate analysis, we controlled for potential reporting bias by place of interview and post-partum length.

\section{Data analysis}

\section{Quantitative analysis}

First, we conducted descriptive analysis to examine the characteristics of the sample and to assess the prevalence of birth companionship and CSLD, as well the 
Table 1 Description of Birth Companionship variables

\begin{tabular}{|c|c|}
\hline Variable & Description \\
\hline Availability of a companion & $\begin{array}{l}\text { Whether or not someone accompanied the woman from home to the health facility. In addition, } \\
\text { we asked if this person stayed with them during labor, delivery, and/or after delivery. }\end{array}$ \\
\hline Type of companion & $\begin{array}{l}\text { Relationship to the person who accompanied them to the facility. Women were allowed to mention } \\
\text { more than one person, and thus, the preferred companion analysis is based on whether or not a } \\
\text { particular type of person was mentioned. }\end{array}$ \\
\hline Allowed continuous support during labor & $\begin{array}{l}\text { Whether or not they were allowed to have someone they wanted to stay with them during labor. A } \\
\text { response of "yes, most of the time," or "yes, all the time" is coded as being allowed continuous labor } \\
\text { support, and "no, never" or "yes, a few times" is coded as not being allowed continuous labor support. }\end{array}$ \\
\hline Allowed continuous support during delivery & $\begin{array}{l}\text { Whether they were allowed to have someone they wanted to stay with them during delivery. } \\
\text { Recoded similarly to "allowed continuous support during labor" }\end{array}$ \\
\hline $\begin{array}{l}\text { Desire for companionship during labor, } \\
\text { delivery, and after delivery }\end{array}$ & $\begin{array}{l}\text { Whether or not they wanted the person who accompanied them to stay with them during labor, } \\
\text { delivery, and/or after delivery; and if they will want a companion at each stage if they were to have } \\
\text { another baby in the future }\end{array}$ \\
\hline Preferred type of companion & $\begin{array}{l}\text { The type of person they would want as a companion during labor, delivery, and/or after delivery if } \\
\text { they were to have another baby. They could mention more than one person, so responses refer to } \\
\text { whether or not they mentioned a particular type of person }\end{array}$ \\
\hline
\end{tabular}

proportion of women who desired companionship and their preferred companions. Next, we used cross tabulations and bivariate logistic regressions to examine the bivariate associations between the dependent and independent variables. We also examined whether the type of companion was associated with being allowed CSLD and women's desire for companionship. Finally, we used multivariate logistic regression to examine the factors associated with being allowed CSLD, net of other factors.

\section{Qualitative analysis}

Using the Braun \& Clarke approach (2006), we analyzed the qualitative data thematically to identify patterns within data [37]. We coded data inductively, considering both the semantic (surface) and latent (underlying) meaning of the text, focusing on salience rather than frequency. We iteratively read and re-read the transcripts and coded line-by-line across the entire data set. Two coders (the first and second authors) double coded half of the transcripts and compared codes to check consistency. We then analyzed initial codes to generate categories and identify themes [37]. We again reviewed transcripts until no new themes emerged. See Additional file 2 for how open codes were used to generate categories and themes. This was however an iterative process with constant reviews and revisions. Throughout the process, we wrote analytic and reflexive memos to capture emerging ideas and examine our assumptions, preconceptions, and reactions to the data. For example, we started the study with the preconception that all women will be unhappy about being denied companions. We were thus surprised by some women's reactions to not being allowed companions and the many reasons they gave for not desiring companions. We therefore wrote a memo on this early on to ensure we adequately captured women's perceptions. We managed data using Atlas.ti. (COREQ checklist in Additional file 3).

\section{Results \\ Results from interviews with women Univariate distributions}

Table 2 shows demographic characteristics of both survey and focus group respondents. Focus group participants were older and more likely to be married, had higher parity, and lower education compared to survey participants. About two-thirds of respondents in both samples belonged to the dominant tribe of the County-the Luo tribe.

The distribution of the birth companionship variables is shown in Table 3 . Among survey respondents, $88 \%$ were accompanied by someone to the health facility. About a third of respondents were accompanied by a partner (29\%) or mother-in-law (28\%). Seventy-eight percent, 26, and $88 \%$ reported the person who accompanied them (subsequently referred to as the companion) stayed with them for some period during labor, delivery, and after delivery respectively. However, only 67 and $29 \%$ of the respondents were allowed continuous support during labor and delivery respectively. Besides, 18,63 , and $6 \%$ did not want their companion to stay with them during labor, delivery, and after delivery respectively. Also, 33, 69, and $10 \%$ reported that if they were to have another baby, they would not want a companion during labor, delivery, and after delivery respectively. For preferred companion in the future, $22 \%$ wanted their partner during labor and $43 \%$ wanted him after delivery, but only $6 \%$ wanted him during the delivery.

\section{Bivariate results}

The bivariate associations between the independent and dependent variables are shown in Tables 4, 5, and 6. We 
Table 2 Demographic characteristics of Study Participants, PQCC Study 2016

\begin{tabular}{llllll}
\hline & \multicolumn{2}{c}{ Survey respondents } & & \multicolumn{2}{c}{ FGD respondents } \\
\cline { 2 - 3 } \cline { 5 - 6 } & No. & $\%$ & & No. & $\%$ \\
\hline Total N & 877 & 100.0 & & 58 & 100
\end{tabular}

Age

$\begin{array}{lllll}15 \text { to } 19 \text { years } & 162 & 18.5 & 4 & 6.9 \\ 20 \text { to } 29 \text { years } & 511 & 58.3 & 38 & 65.5 \\ 30 \text { to } 48 \text { years } & 204 & 23.3 & 16 & 27.6\end{array}$

Marital status

$\begin{array}{lllll}\text { Single } & 140 & 16.0 & 5 & 8.6 \\ \text { Partnered/Cohabiting } & 3 & 0.3 & 1 & 1.7 \\ \text { Married } & 687 & 78.3 & 51 & 87.9 \\ \text { Widowed } & 35 & 4.0 & 1 & 1.7 \\ \text { Divorced/Separated } & 12 & 1.4 & & \end{array}$

Number of births

$\begin{array}{lllll}1.0 & 290 & 33.1 & 6 & 10.3 \\ 2.0 & 185 & 21.1 & 8 & 13.8 \\ 3.0 & 163 & 18.6 & 11 & 19 \\ 4 \text { or more } & 239 & 27.3 & 33 & 56.9\end{array}$

Education

$\begin{array}{lcccc}\text { No school/Primary } & 495 & 56.4 & 46 & 79.3 \\ \begin{array}{l}\text { Post-primary/Nocational/ } \\ \text { Secondary }\end{array} & 271 & 30.9 & 10 & 17.2 \\ \text { College or above } & 111 & 12.7 & 2 & 3.4\end{array}$

Literacy: writing

No, cannot write
Yes, but with some difficulty
Yes, Very well

$\begin{array}{llll}31 & 3.5 & 1 & 1.7\end{array}$

$\begin{array}{llll}141 & 16.1 & 38 & 65.5\end{array}$

Literacy: reading

No, cannot read
Yes, but with some difficulty
Yes, Very well

Employed

$\begin{array}{lllll}\text { No } & 658 & 75.0 & 39 & 67.2 \\ \text { Yes } & 219 & 25.0 & 19 & 32.8\end{array}$

Wealth Quintile

$\begin{array}{lll}\text { Poorest } & 190 & 21.7 \\ \text { Poorer } & 190 & 21.7 \\ \text { Middle } & 135 & 15.4 \\ \text { Richer } & 172 & 19.6 \\ \text { Richest } & 190 & 21.7\end{array}$

Delivery facility type

$\begin{array}{lllll}\text { Gov't Hospital } & 404 & 46.1 & 27 & 49.1 \\ \text { Gov't Health Center } & 362 & 41.3 & 23 & 41.8 \\ \text { Mission/Private facility } & 111 & 12.7 & 5 & 9.1\end{array}$

Delivery provider
Table 2 Demographic characteristics of Study Participants, PQCC Study 2016 (Continued)

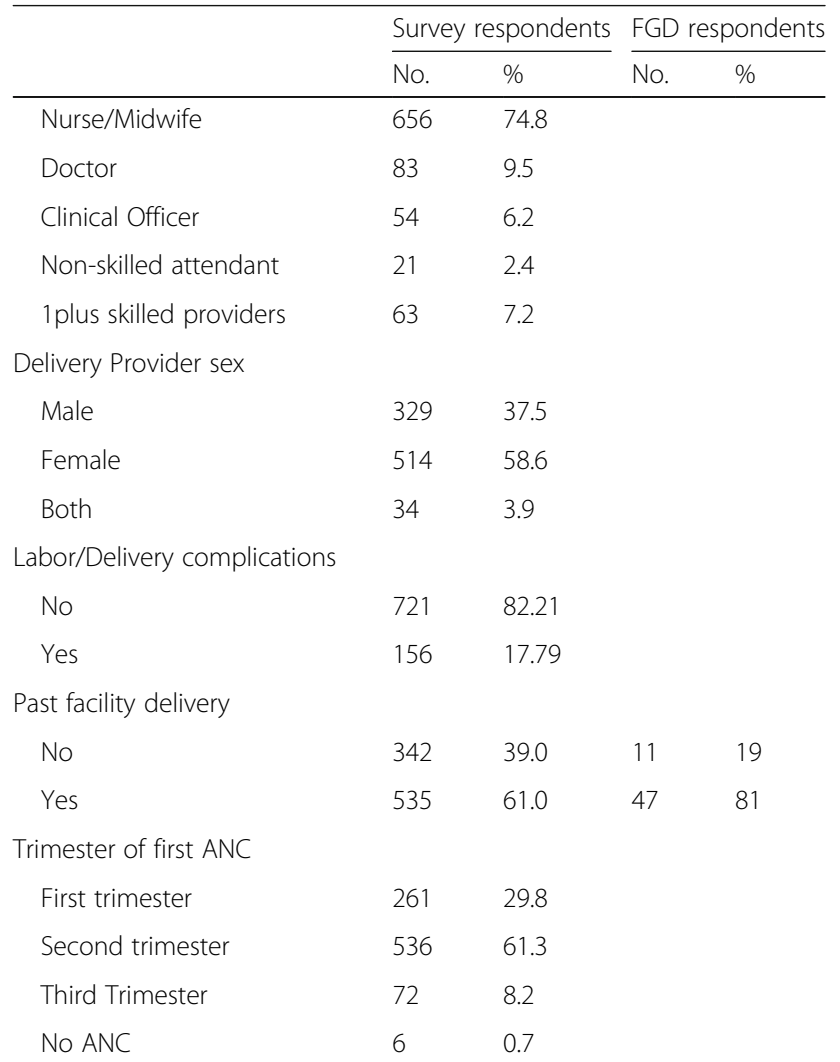

Number of ANC visits

$\begin{array}{lllll}\text { No ANC } & 6 & 0.7 & & \\ \text { Less than 4 } & 281 & 32.2 & & \\ 4 \text { or } 5 & 485 & 55.6 & & \\ 6 \text { plus } & 100 & 11.5 & & \\ \text { Tribe } & & & & \\ \text { Luo } & 584 & 66.6 & 38 & 65.5 \\ \text { Kuria } & 208 & 23.7 & 12 & 20.7 \\ \text { Other } & 85 & 9.7 & 8 & 13.8\end{array}$

Religion

\begin{tabular}{|c|c|c|c|c|}
\hline Catholic & 242 & 27.6 & 12 & 20.7 \\
\hline Protestant/Pentecostal & 191 & 21.8 & 22 & 37.9 \\
\hline Seventh Day Adventist & 263 & 30.0 & 14 & 24.1 \\
\hline Other Christian & 166 & 18.9 & 7 & 12.1 \\
\hline Muslim/other religion & 15 & 1.7 & 3 & 5.2 \\
\hline \multicolumn{5}{|l|}{ ostpartum length } \\
\hline Less than 1 week & 75 & 8.6 & 1 & 1.7 \\
\hline 1 week or more & 802 & 91.4 & 57 & 98.3 \\
\hline \multicolumn{5}{|l|}{ Place of Interview } \\
\hline Health facility & 356 & 40.6 & 10 & 17.2 \\
\hline In the community/a home & 521 & 59.4 & 48 & 82.8 \\
\hline
\end{tabular}


Table 3 Distribution of Birth Companionship variables, PQCC Study 2016

\begin{tabular}{|c|c|c|c|c|c|c|}
\hline Birth Companionship Variables & No. & $\%$ & No. & $\%$ & No. & $\%$ \\
\hline \multicolumn{7}{|l|}{ Accompanied from home to facility } \\
\hline No & 106 & 12.4 & & & & \\
\hline Yes & 751 & 87.6 & & & & \\
\hline \multicolumn{7}{|l|}{ Accompanied by } \\
\hline Partner-Husband & 251 & 29.3 & & & & \\
\hline Mother-in-law & 236 & 27.5 & & & & \\
\hline Mother & 100 & 11.7 & & & & \\
\hline Sister/Sister-in-law & 164 & 19.1 & & & & \\
\hline Friend/Neighbor & 112 & 13.1 & & & & \\
\hline Other $^{a}$ & 74 & 8.7 & & & & \\
\hline Companion stayed during & \multicolumn{2}{|l|}{ Labor } & \multicolumn{2}{|c|}{ Delivery } & \multicolumn{2}{|c|}{ Post-delivery } \\
\hline No & 157 & 20.9 & 556 & 74 & 86 & 11.5 \\
\hline Yes & 588 & 78.3 & 192 & 25.6 & 661 & 88 \\
\hline Don't know & 6 & 0.8 & 3 & 0.4 & 4 & 0.5 \\
\hline Companion allowed during & \multicolumn{2}{|l|}{ Labor } & \multicolumn{2}{|c|}{ Delivery } & & \\
\hline No, never & 162 & 18.9 & 524 & 61.1 & & \\
\hline Yes, a few times & 103 & 12 & 72 & 8.4 & & \\
\hline Yes, most of the time & 230 & 26.8 & 110 & 12.8 & & \\
\hline Yes, all the time & 357 & 41.7 & 138 & 16.1 & & \\
\hline Wanted Companion to stay during & \multicolumn{2}{|l|}{ Labor } & \multicolumn{2}{|c|}{ Delivery } & \multicolumn{2}{|c|}{ Post-delivery } \\
\hline No & 138 & 18.4 & 476 & 63.4 & 48 & 6.4 \\
\hline Yes & 608 & 81 & 267 & 35.6 & 701 & 93.3 \\
\hline Don't know & 5 & 0.7 & 8 & 1.1 & 2 & 0.3 \\
\hline Will want companion in future during & \multicolumn{2}{|l|}{ Labor } & \multicolumn{2}{|c|}{ Delivery } & \multicolumn{2}{|c|}{ Post-delivery } \\
\hline No & 285 & 33.3 & 592 & 69.1 & 85 & 9.9 \\
\hline Yes & 560 & 65.3 & 254 & 29.6 & 761 & 88.8 \\
\hline Don't know & 12 & 1.4 & 11 & 1.3 & 11 & 1.3 \\
\hline Preferred companion type in future during & \multicolumn{2}{|l|}{ Labor } & \multicolumn{2}{|c|}{ Delivery } & \multicolumn{2}{|c|}{ Post-delivery } \\
\hline Partner-Husband & 188 & 21.9 & 49 & 5.7 & 370 & 43.2 \\
\hline Mother in Law & 234 & 27.3 & 119 & 13.9 & 362 & 42.2 \\
\hline Mother & 91 & 10.6 & 42 & 4.9 & 138 & 16.1 \\
\hline Sister/Sister in law & 165 & 19.3 & 63 & 7.4 & 244 & 28.5 \\
\hline Friend/Neighbor & 77 & 9 & 31 & 3.6 & 159 & 18.6 \\
\hline Community Health Volunteer & 4 & 0.5 & 4 & 0.5 & 8 & 0.9 \\
\hline Nurse/Midwife & 35 & 4.1 & 21 & 2.5 & 49 & 5.7 \\
\hline Doctor & 10 & 1.2 & 10 & 1.2 & 14 & 1.6 \\
\hline Other & 23 & 2.7 & 8 & 0.9 & 26 & 3 \\
\hline \multicolumn{7}{|l|}{ Acceptability of being denied a companion } \\
\hline Unacceptable in all instances & 722 & 84.2 & & & & \\
\hline Acceptable in certain instances & 112 & 13.1 & & & & \\
\hline Acceptable in all instances & 23 & 2.7 & & & & \\
\hline Total & 857 & 100 & 857 & 100 & 857 & 100 \\
\hline
\end{tabular}

${ }^{a}$ Other includes co-wives, grand-mother, community health volunteers, etc. 
Table 4 Bivariate distribution of selected birth companionship variables by demographic and facility characteristics, PQCC Study 2016

\begin{tabular}{|c|c|c|c|c|c|c|}
\hline & \multirow{2}{*}{\multicolumn{2}{|c|}{ Accompanied to facility }} & \multicolumn{4}{|c|}{ Allowed continuous support during } \\
\hline & & & \multicolumn{2}{|c|}{ Labor } & \multicolumn{2}{|c|}{ Delivery } \\
\hline & No. & $\%$ & No. & $\%$ & No. & $\%$ \\
\hline \multicolumn{7}{|l|}{ Age } \\
\hline 15 to 19 years & 156 & $96.3^{* *}$ & 118 & 72.8 & 58 & 35.8 \\
\hline 20 to 29 years & 442 & 86.5 & 352 & 69.2 & 153 & 29.9 \\
\hline 30 to 48 years & 172 & 84.3 & 130 & 63.7 & 56 & 27.5 \\
\hline \multicolumn{7}{|l|}{ Marital Status } \\
\hline Not married & 172 & 90.5 & 137 & 72.1 & 69 & $36.3^{*}$ \\
\hline Currently married & 598 & 87 & 463 & 67.6 & 198 & 28.8 \\
\hline \multicolumn{7}{|l|}{ Number of births } \\
\hline 1.0 & 274 & $94.5^{* * *}$ & 216 & $74.5^{* *}$ & 102 & 35.2 \\
\hline 2.0 & 163 & 88.1 & 134 & 73.2 & 51 & 27.6 \\
\hline 3.0 & 137 & 84 & 103 & 63.2 & 53 & 32.5 \\
\hline 4 or more & 196 & 82 & 147 & 61.5 & 61 & 25.5 \\
\hline \multicolumn{7}{|l|}{ Education } \\
\hline No school/Primary & 425 & 85.9 & 323 & $65.4^{*}$ & 132 & $26.7^{*}$ \\
\hline Post-primary/vocational/Secondary & 246 & 90.8 & 189 & 70 & 99 & 36.5 \\
\hline College or above & 99 & 89.2 & 88 & 79.3 & 36 & 32.4 \\
\hline \multicolumn{7}{|l|}{ Literacy } \\
\hline No, cannot write & 28 & 90.3 & 13 & $41.9^{* * *}$ & 7 & $22.6^{* * *}$ \\
\hline Yes, but with some difficulty & 122 & 86.5 & 84 & 59.6 & 20 & 14.2 \\
\hline Yes, Very well & 620 & 87.9 & 503 & 71.6 & 240 & 34 \\
\hline \multicolumn{7}{|l|}{ Household wealth } \\
\hline Poorest/poorer & 332 & 87.4 & 236 & $62.3^{* *}$ & 105 & $27.6^{*}$ \\
\hline Middle & 122 & 90.4 & 93 & 68.9 & 37 & 27.4 \\
\hline Richer/richest & 316 & 87.3 & 271 & 75.1 & 125 & 34.5 \\
\hline \multicolumn{7}{|l|}{ Employed } \\
\hline No & 576 & 87.5 & 427 & $65.1^{* * *}$ & 198 & 30.1 \\
\hline Yes & 194 & 88.6 & 173 & 79 & 69 & 31.5 \\
\hline \multicolumn{7}{|l|}{ Pregnancy complication } \\
\hline No & 431 & 87.2 & 349 & 70.8 & 168 & $34^{* *}$ \\
\hline Yes & 339 & 88.5 & 251 & 65.7 & 99 & 25.8 \\
\hline \multicolumn{7}{|l|}{ Birth Complication } \\
\hline No & 633 & 87.8 & 515 & $71.6^{* * *}$ & 242 & $33.6^{* * *}$ \\
\hline Yes & 137 & 87.8 & 85 & 54.5 & 25 & 16 \\
\hline \multicolumn{7}{|l|}{ Past health facility delivery } \\
\hline No & 315 & $92.1^{* *}$ & 242 & 70.8 & 117 & 34.2 \\
\hline Yes & 455 & 85 & 358 & 67.2 & 150 & 28 \\
\hline \multicolumn{7}{|l|}{ ANC visits } \\
\hline Less than 4 & 240 & $83.6^{* *}$ & 200 & 69.7 & 92 & 32.1 \\
\hline 4 plus & 526 & 89.9 & 396 & 67.8 & 173 & 29.6 \\
\hline \multicolumn{7}{|l|}{ Tribe } \\
\hline Luo & 496 & $84.9^{* * *}$ & 426 & $73.1^{* * *}$ & 189 & 32.4 \\
\hline
\end{tabular}


Table 4 Bivariate distribution of selected birth companionship variables by demographic and facility characteristics, PQCC Study 2016 (Continued)

\begin{tabular}{|c|c|c|c|c|c|c|}
\hline & \multirow{2}{*}{\multicolumn{2}{|c|}{ Accompanied to facility }} & \multicolumn{4}{|c|}{ Allowed continuous support during } \\
\hline & & & \multicolumn{2}{|c|}{ Labor } & \multicolumn{2}{|c|}{ Delivery } \\
\hline & No. & $\%$ & No. & $\%$ & No. & $\%$ \\
\hline Kuria & 200 & 96.2 & 117 & 56.5 & 53 & 25.5 \\
\hline Other & 74 & 87.1 & 57 & 67.1 & 25 & 29.4 \\
\hline \multicolumn{7}{|l|}{ Religion } \\
\hline Catholic & 215 & 88.8 & 175 & 72.6 & 68 & 28.1 \\
\hline Protestant/Pentecostal & 165 & 86.4 & 122 & 63.9 & 53 & 27.7 \\
\hline Seventh Day Adventist & 236 & 89.7 & 182 & 69.5 & 94 & 35.7 \\
\hline Other Christian & 142 & 85.5 & 112 & 67.5 & 46 & 27.7 \\
\hline Muslim/other religion & 12 & 80 & 9 & 60 & 6 & 40 \\
\hline \multicolumn{7}{|l|}{ Delivery facility type } \\
\hline Gov't Hospital & 369 & $91.3^{* *}$ & 257 & $63.9^{* *}$ & 115 & 28.5 \\
\hline Gov't Health Center & 303 & 83.7 & 273 & 75.4 & 114 & 31.5 \\
\hline Mission/Private facility & 98 & 88.3 & 70 & 63.1 & 38 & 34.2 \\
\hline \multicolumn{7}{|l|}{ Delivery provider } \\
\hline Nurse/Midwife & 578 & 88.1 & 462 & $70.6^{* *}$ & 209 & 31.9 \\
\hline Doctor/Clinical Officer & 119 & 86.9 & 89 & 65 & 40 & 29.2 \\
\hline Non-skilled attendant & 17 & 81 & 17 & 81 & 8 & 38.1 \\
\hline 1 plus skilled providers & 56 & 88.9 & 32 & 50.8 & 10 & 15.9 \\
\hline \multicolumn{7}{|l|}{ Delivery Provider sex } \\
\hline Male & 294 & 89.4 & 206 & $62.6^{*}$ & 82 & $24.9^{*}$ \\
\hline Female & 445 & 86.6 & 370 & 72.3 & 173 & 33.7 \\
\hline Both & 31 & 91.2 & 24 & 70.6 & 12 & 35.3 \\
\hline \multicolumn{7}{|l|}{ Accompanied by } \\
\hline \multicolumn{7}{|l|}{ Partner } \\
\hline No & & & 434 & 69.7 & 208 & $33.3^{* *}$ \\
\hline Yes & & & 166 & 65.9 & 59 & 23.3 \\
\hline \multicolumn{7}{|l|}{ Mother-in-law } \\
\hline No & & & 442 & 70 & 186 & 29.4 \\
\hline Yes & & & 158 & 64.8 & 81 & 33.1 \\
\hline \multicolumn{7}{|l|}{ Mother } \\
\hline No & & & 525 & 67.9 & 231 & 29.8 \\
\hline Yes & & & 75 & 73.5 & 36 & 35.3 \\
\hline \multicolumn{7}{|l|}{ Sister/Sister in law } \\
\hline No & & & 465 & $65.7^{* * *}$ & 223 & 31.4 \\
\hline Yes & & & 135 & 80.8 & 44 & 26.3 \\
\hline \multicolumn{7}{|l|}{ Friend/Neighbor } \\
\hline No & & & 519 & 68.2 & 238 & 31.2 \\
\hline Yes & & & 81 & 71.1 & 29 & 25.4 \\
\hline \multicolumn{7}{|l|}{ Facility crowded } \\
\hline No or a few times & & & 425 & $72.2^{* *}$ & 172 & 29.1 \\
\hline Most or all the time & & & 173 & 60.9 & 94 & 33.1 \\
\hline
\end{tabular}


Table 4 Bivariate distribution of selected birth companionship variables by demographic and facility characteristics, PQCC Study 2016 (Continued)

\begin{tabular}{|c|c|c|c|c|c|c|}
\hline & \multirow{2}{*}{\multicolumn{2}{|c|}{ Accompanied to facility }} & \multicolumn{4}{|c|}{ Allowed continuous support during } \\
\hline & & & \multicolumn{2}{|c|}{ Labor } & \multicolumn{2}{|c|}{ Delivery } \\
\hline & No. & $\%$ & No. & $\%$ & No. & $\%$ \\
\hline \multicolumn{7}{|c|}{ Desired labor companion } \\
\hline No & & & 68 & $47.9^{* * *}$ & & \\
\hline Yes & & & 459 & 73.9 & & \\
\hline Don't know & & & 3 & 60 & & \\
\hline \multicolumn{7}{|c|}{ Desired delivery companion } \\
\hline No & & & & & 92 & $18.8^{* * *}$ \\
\hline Yes & & & & & 135 & 49.6 \\
\hline Don't know & & & & & 4 & 50 \\
\hline Total & 770 & 87.8 & 600 & 68.6 & 267 & 30.4 \\
\hline
\end{tabular}

find that younger and primiparous women who had no past facility delivery, as well as women from the Kuria tribe, women with four or more antenatal visits, and women who delivered in higher level facilities were more likely (than their reference groups) to be accompanied to the health facility (Table 4).

Continuous labor and delivery support Younger, wealthier, employed, college educated, and literate women were more likely to be allowed continuous labor support. On the other hand, women who were of the Kuria tribe, had birth complications, reported the facility was over crowded most of the time, delivered in hospitals, and those who did not want their companion to stay with them, were less likely to be allowed continuous labor support. Many of the factors associated with continuous delivery support in bivariate analysis are similar to those for continuous labor support (Table 4).

Desire for labor and delivery support Employed women and women accompanied by a sister or sister-in-law were more likely to desire companionship during labor. In addition, younger, unmarried, primiparous, literate, and wealthier women, as well as those accompanied by a mother or a non-relative were more likely to desire companionship at the time of delivery. Women who were accompanied by a partner were less likely to desire companionship during labor and delivery. Also, women who perceived the health facility to be over crowded most of the time were less likely to desire labor companionship. Women who were allowed CSLD at their last birth were more likely to desire it in the future (Table 5).

Preferred companion Wealthier and more educated women were more likely to be accompanied by their partners and to desire labor companionship from their partners in the future. They were also less likely to be accompanied by their mothers-in-law and to desire companionship from their mothers-in-law in the future. Kuria women were more likely to be accompanied by their mothers-in-law and to desire companionship from their mothers-in-law during labor in the future (Table 6).

\section{Multivariate results}

Table 7 shows multivariate logistic regressions of continuous labor and delivery support on various predictors. The multivariate results for desire for companionship and preference for male partners are shown in Additional file 4.

Continuous labor support Wealthier, employed, and literate women had close to two times higher odds of being allowed continuous labor support than the poorest, unemployed, and illiterate women respectively; and Kuria women had about $40 \%$ lower odds of being allowed continuous labor support than Luo women. Also, those who delivered in a health center and were accompanied by a sister or sister-in-law had close to two times higher odds of being allowed continuous labor support than those who delivered in hospitals and those not accompanied by a sister or sister-in-law respectively. Women who desired a labor companion had about $40 \%$ higher odds of being allowed continuous labor support than those who did not desire one. Additionally, women who reported the facility was over crowded most or all the time had about 35\% lower odds of being allowed continuous labor support than those who said it was not crowded or crowded only a few times.

Continuous delivery support Women had lower odds of being allowed continuous delivery support if they had a birth complication and if there were two or more skilled providers present at the delivery than if they had 
Table 5 Bivariate distribution of selected birth companionship variables by demographic and facility characteristics, PQCC study 2016

\begin{tabular}{|c|c|c|c|c|c|c|c|c|}
\hline & \multicolumn{4}{|c|}{ Desire for labor support } & \multicolumn{4}{|c|}{ Desire for delivery support } \\
\hline & \multicolumn{2}{|c|}{ Last birth } & \multicolumn{2}{|c|}{ Future birth } & \multicolumn{2}{|c|}{ Last birth } & \multicolumn{2}{|c|}{ Future birth } \\
\hline & No. & $\%$ & No. & $\%$ & No. & $\%$ & No. & $\%$ \\
\hline \multicolumn{9}{|l|}{ Age } \\
\hline 15 to 19 years & 128 & 82.1 & 108 & $66.7^{*}$ & 73 & $46.8^{*}$ & 58 & 35.8 \\
\hline 20 to 29 years & 352 & 79.6 & 327 & 64 & 144 & 32.6 & 137 & 26.9 \\
\hline 30 to 48 years & 142 & 82.6 & 139 & 68.1 & 55 & 32 & 62 & 30.4 \\
\hline \multicolumn{9}{|l|}{ Marital Status } \\
\hline Not married & 149 & 86.6 & 125 & $65.8^{* *}$ & 81 & $47.1^{* *}$ & 65 & $34.2^{*}$ \\
\hline Currently married & 473 & 79.1 & 449 & 65.4 & 191 & 31.9 & 192 & 28 \\
\hline \multicolumn{9}{|l|}{ Number of births } \\
\hline 1.0 & 233 & 85 & 190 & 65.5 & 122 & $44.5^{* *}$ & 108 & $37.2^{*}$ \\
\hline 2.0 & 134 & 82.2 & 132 & 71.4 & 55 & 33.7 & 51 & 27.7 \\
\hline 3.0 & 105 & 76.6 & 103 & 63.2 & 38 & 27.7 & 37 & 22.7 \\
\hline 4 or more & 150 & 76.5 & 149 & 62.3 & 57 & 29.1 & 61 & 25.5 \\
\hline \multicolumn{9}{|l|}{ Education } \\
\hline No school/Primary & 336 & 79.1 & 321 & 64.8 & 137 & 32.2 & 141 & 28.5 \\
\hline Post-primary/Nocational/Secondary & 202 & 82.1 & 182 & 67.2 & 99 & 40.2 & 78 & 28.8 \\
\hline College or above & 84 & 84.8 & 71 & 64 & 36 & 36.4 & 38 & 34.2 \\
\hline \multicolumn{9}{|l|}{ Literacy } \\
\hline No, cannot write & 22 & 78.6 & 22 & 71 & 9 & $32.1^{*}$ & 6 & 19.4 \\
\hline Yes, but with some difficulty & 94 & 77 & 98 & 69.5 & 28 & 23 & 38 & 27 \\
\hline Yes, Very well & 506 & 81.6 & 454 & 64.4 & 235 & 37.9 & 213 & 30.3 \\
\hline \multicolumn{9}{|l|}{ Household wealth } \\
\hline Poorest/poorer & 260 & 78.3 & 251 & 66.1 & 103 & $31^{* *}$ & 104 & 27.4 \\
\hline Middle & 98 & 80.3 & 86 & 63.7 & 39 & 32 & 33 & 24.4 \\
\hline Richer/richest & 264 & 83.5 & 237 & 65.5 & 130 & 41.1 & 120 & 33.1 \\
\hline \multicolumn{9}{|l|}{ Employed } \\
\hline No & 452 & $78.5^{*}$ & 402 & $61.1^{* * *}$ & 200 & 34.7 & 184 & 28 \\
\hline Yes & 170 & 87.6 & 172 & 78.5 & 72 & 37.1 & 73 & 33.3 \\
\hline \multicolumn{9}{|l|}{ Pregnancy complication } \\
\hline No & 346 & 80.3 & 309 & $62.6^{*}$ & 149 & 34.6 & 144 & 29.1 \\
\hline Yes & 276 & 81.4 & 265 & 69.2 & 123 & 36.3 & 113 & 29.6 \\
\hline \multicolumn{9}{|l|}{ Birth Complication } \\
\hline No & 517 & 81.7 & 468 & 64.9 & 224 & 35.4 & 210 & 29.2 \\
\hline Yes & 105 & 76.6 & 106 & 67.9 & 48 & 35 & 47 & 30.1 \\
\hline \multicolumn{9}{|l|}{ Past health facility delivery } \\
\hline No & 265 & 84.1 & 225 & 65.8 & 140 & $44.4^{* * *}$ & 122 & $35.7^{* *}$ \\
\hline Yes & 357 & 78.5 & 349 & 65.2 & 132 & 29 & 135 & 25.3 \\
\hline \multicolumn{9}{|l|}{ ANC visits } \\
\hline Less than 4 & 196 & 81.7 & 190 & 66.2 & 86 & 35.8 & 91 & 31.8 \\
\hline 4 plus & 423 & 80.4 & 380 & 65 & 183 & 34.8 & 163 & 27.9 \\
\hline \multicolumn{9}{|l|}{ Tribe } \\
\hline Luo & 407 & 82.1 & 370 & 63.4 & 167 & 33.7 & 143 & $24.5^{* * *}$ \\
\hline
\end{tabular}


Table 5 Bivariate distribution of selected birth companionship variables by demographic and facility characteristics, PQCC study 2016 (Continued)

\begin{tabular}{|c|c|c|c|c|c|c|c|c|}
\hline & \multicolumn{4}{|c|}{ Desire for labor support } & \multicolumn{4}{|c|}{ Desire for delivery support } \\
\hline & \multicolumn{2}{|c|}{ Last birth } & \multicolumn{2}{|c|}{ Future birth } & \multicolumn{2}{|c|}{ Last birth } & \multicolumn{2}{|c|}{ Future birth } \\
\hline & No. & $\%$ & No. & $\%$ & No. & $\%$ & No. & $\%$ \\
\hline Kuria & 160 & 80 & 146 & 70.2 & 71 & 35.5 & 79 & 38 \\
\hline Other & 55 & 74.3 & 58 & 68.2 & 34 & 45.9 & 35 & 41.2 \\
\hline \multicolumn{9}{|l|}{ Religion } \\
\hline Catholic & 180 & 83.7 & 155 & 64 & 73 & 34 & 72 & $29.8^{* *}$ \\
\hline Protestant/Pentecostal & 129 & 78.2 & 125 & 65.4 & 72 & 43.6 & 77 & 40.5 \\
\hline Seventh Day Adventist & 196 & 83.1 & 184 & 70 & 79 & 33.5 & 73 & 27.8 \\
\hline Other Christian & 108 & 76.1 & 102 & 61.4 & 44 & 31 & 33 & 19.9 \\
\hline Muslim/other religion & 9 & 75 & 8 & 53.3 & 4 & 33.3 & 2 & 13.3 \\
\hline \multicolumn{9}{|l|}{ Delivery facility type } \\
\hline Gov't Hospital & 294 & 79.7 & 256 & 63.4 & 133 & 36 & 130 & 32.3 \\
\hline Gov't Health Center & 251 & 82.8 & 248 & 68.5 & 100 & 33 & 89 & 24.6 \\
\hline Mission/Private facility & 77 & 78.6 & 70 & 63.1 & 39 & 39.8 & 38 & 34.2 \\
\hline \multicolumn{9}{|l|}{ Delivery provider } \\
\hline Nurse/Midwife & 472 & 81.7 & 438 & 66.8 & 211 & 36.5 & 203 & 31 \\
\hline Doctor/Clinical Officer & 94 & 79 & 84 & 61.3 & 35 & 29.4 & 29 & 21.2 \\
\hline Non-skilled attendant & 13 & 76.5 & 14 & 66.7 & 6 & 35.3 & 6 & 28.6 \\
\hline 1 plus skilled providers & 43 & 76.8 & 38 & 60.3 & 20 & 35.7 & 19 & 30.2 \\
\hline \multicolumn{9}{|l|}{ Delivery Provider sex } \\
\hline Male & 235 & 79.9 & 215 & 65.3 & 108 & 36.7 & 104 & 31.6 \\
\hline Female & 363 & 81.6 & 337 & 65.6 & 152 & 34.2 & 141 & 27.5 \\
\hline Both & 24 & 77.4 & 22 & 64.7 & 12 & 38.7 & 12 & 35.3 \\
\hline \multicolumn{9}{|l|}{ Accompanied by } \\
\hline \multicolumn{9}{|l|}{ Partner } \\
\hline No & 443 & $85.7^{* * *}$ & 412 & 66 & 209 & $40.4^{* * *}$ & 195 & $31.3^{*}$ \\
\hline Yes & 179 & 70.8 & 162 & 64 & 63 & 24.9 & 62 & 24.5 \\
\hline \multicolumn{9}{|l|}{ Mother-in-law } \\
\hline No & 419 & 79.8 & 401 & 63.4 & 180 & 34.3 & 168 & $26.6^{*}$ \\
\hline Yes & 203 & 82.9 & 173 & 70.6 & 92 & 37.6 & 89 & 36.5 \\
\hline \multicolumn{9}{|l|}{ Mother } \\
\hline No & 534 & 79.9 & 504 & $65^{*}$ & 224 & $33.5^{*}$ & 221 & 28.6 \\
\hline Yes & 88 & 86.3 & 70 & 68.6 & 48 & 47.1 & 36 & 35.3 \\
\hline \multicolumn{9}{|l|}{ Sister/Sister in law } \\
\hline No & 476 & $78.9^{*}$ & 456 & 64.2 & 212 & 35.2 & 206 & 29.1 \\
\hline Yes & 146 & 87.4 & 118 & 70.7 & 60 & 35.9 & 51 & 30.5 \\
\hline \multicolumn{9}{|l|}{ Friend/Neighbor } \\
\hline No & 532 & 81.1 & 504 & 66.1 & 222 & $33.8^{*}$ & 222 & 29.1 \\
\hline Yes & 90 & 78.9 & 70 & 61.4 & 50 & 43.9 & 35 & 30.7 \\
\hline \multicolumn{9}{|l|}{ Facility crowded } \\
\hline No or a few times & 428 & $83.4^{*}$ & 400 & 67.7 & 185 & 36.1 & 174 & 29.4 \\
\hline Most or all the time & 192 & 75.3 & 172 & 60.6 & 86 & 33.7 & 82 & 28.9 \\
\hline
\end{tabular}


Table 5 Bivariate distribution of selected birth companionship variables by demographic and facility characteristics, PQCC study 2016 (Continued)

\begin{tabular}{|c|c|c|c|c|c|c|c|c|}
\hline & \multicolumn{4}{|c|}{ Desire for labor support } & \multicolumn{4}{|c|}{ Desire for delivery support } \\
\hline & \multicolumn{2}{|c|}{ Last birth } & \multicolumn{2}{|c|}{ Future birth } & \multicolumn{2}{|c|}{ Last birth } & \multicolumn{2}{|c|}{ Future birth } \\
\hline & No. & $\%$ & No. & $\%$ & No. & $\%$ & No. & $\%$ \\
\hline \multicolumn{9}{|c|}{ Allowed continuous labor support } \\
\hline No or a few times & 162 & $68.1^{* * *}$ & 146 & $53.1^{* * *}$ & & & & \\
\hline Most or all the time & 459 & 86.6 & 428 & 71.3 & & & & \\
\hline \multicolumn{9}{|c|}{ Allowed continuous delivery support } \\
\hline No or a few times & & & & & 137 & $25.4^{* * *}$ & 139 & $22.8^{* * *}$ \\
\hline Most or all the time & & & & & 135 & 58.4 & 118 & 44.2 \\
\hline Total & 622 & 80.8 & 574 & 65.5 & 272 & 35.3 & 257 & 29.3 \\
\hline
\end{tabular}

${ }^{*} p<0.05 * * p<0.01 * * * 0<0.001$

no complication and were assisted by only a nurse or midwife respectively. In spite of the fact that there was no significant association between being allowed continuous delivery support and perception of overcrowding in the bivariate analysis, this association became significant in the multivariate model, but in an unexpected direction-women who reported that the health facility was overcrowded most or all the time had about $69 \%$ higher odds of being allowed continuous delivery support than those who perceived that the facility was not overcrowded. Also, women accompanied by a partner had about $40 \%$ lower odds of being allowed continuous delivery support than those not accompanied by a partner; and those who desired a companion at delivery had close to two times higher odds of being allowed continuous delivery support.

\section{Women's perspectives on labor and delivery support: Qualitative data from FGDs with women}

The overarching themes from the analysis of the data from FGD with women are on their feelings about being allowed or denied a companion during labor and delivery, reasons why they do or do not want a companion, and their preferences for different types of companions.

\section{Feelings about being allowed or denied a companion during labor and delivery}

Most women were happy when their companions were allowed to stay with them during labor, and unhappy when they were not allowed to stay with them.

"They allowed the relatives to be with us ....I felt good because there are times you can be going to bath, and so they will remain and take care of the baby for you." (KP5)

"No they didn't [allow relative to stay] and I did not like it because they are busy with their own things and it is this relative of yours who can help you with your problem..." (WP1)
Companions were however only allowed at certain times, like during labor but not at the time of delivery, or only after the baby was born; or during certain times of the day like at night, but not in the morning. Many felt it was inappropriate to not be allowed a companion during labor and after delivery.

"I wanted her to be with me so that I can explain to her how am feeling so that in case I need help then I send her to go and call the doctor or nurse to come and help me quickly and they kept on telling them that "get out care takers your time is not now, wait until they give birth is when you can come in", and yet after giving birth am not as serious as when I still have my labor pains when am not supposed to be alone and so I felt bad." (WP2)

However, many did not mind not being allowed a companion at the time of delivery for various reasons described in later sections.

"I felt it was good if they were allowed to be with me after having my baby. But before, it was good that they were not allowed to be with me." (CP10)

It appeared some providers did not take time to explain to women about why they were not allowed a birth companion, and were sometimes abusive when disallowing the companion.

"They refused and even chased the person who accompanied me and told her leave her alone .... you did not give her the pregnancy she will deliver it herself'... that was not good." (MP2)

Women therefore inferred various reasons why their companions were not allowed to stay with them. Some felt that it was a facility requirement that applied to 
Table 6 Bivariate distribution of selected birth companionship variables by demographic and facility characteristics, PQCC Study 2016

\begin{tabular}{|c|c|c|c|c|c|c|}
\hline & \multicolumn{3}{|c|}{ Accompanied by Partner } & \multicolumn{3}{|c|}{ Accompanied by mother-in-law } \\
\hline & No. & $\%$ & $p$-value & No. & $\%$ & $p$-value \\
\hline \multicolumn{7}{|l|}{ Age } \\
\hline 15 to 19 years & 26 & 16 & $* * *$ & 43 & 26.5 & \\
\hline 20 to 29 years & 154 & 30.1 & & 150 & 29.4 & \\
\hline 30 to 48 years & 73 & 35.8 & & 52 & 25.5 & \\
\hline \multicolumn{7}{|l|}{ Marital Status } \\
\hline Not married & 12 & 6.3 & $* * *$ & 21 & 11.1 & $* * *$ \\
\hline Currently married & 241 & 35.1 & & 224 & 32.6 & \\
\hline \multicolumn{7}{|l|}{ Number of births } \\
\hline 1.0 & 57 & 19.7 & $* * *$ & 67 & 23.1 & * \\
\hline 2.0 & 70 & 37.8 & & 62 & 33.5 & \\
\hline 3.0 & 50 & 30.7 & & 53 & 32.5 & \\
\hline 4 or more & 76 & 31.8 & & 63 & 26.4 & \\
\hline \multicolumn{7}{|l|}{ Education } \\
\hline No school/Primary & 117 & 23.6 & $* * *$ & 150 & 30.3 & * \\
\hline Post-primary/vocational/Secondary & 86 & 31.7 & & 76 & 28 & \\
\hline College or above & 50 & 45 & & 19 & 17.1 & \\
\hline \multicolumn{7}{|l|}{ Literacy } \\
\hline No, cannot write & 11 & 35.5 & & 11 & 35.5 & \\
\hline Yes, but with some difficulty & 39 & 27.7 & & 40 & 28.4 & \\
\hline Yes, Very well & 203 & 28.8 & & 194 & 27.5 & \\
\hline \multicolumn{7}{|l|}{ Household wealth } \\
\hline Poorest/poorer & 93 & 24.5 & * & 135 & 35.5 & $* * *$ \\
\hline Middle & 40 & 29.6 & & 33 & 24.4 & \\
\hline Richer/richest & 120 & 33.1 & & 77 & 21.3 & \\
\hline \multicolumn{7}{|l|}{ Employed } \\
\hline No & 176 & 26.7 & * & 177 & 26.9 & \\
\hline Yes & 77 & 35.2 & & 68 & 31.1 & \\
\hline \multicolumn{7}{|l|}{ Has Health Insurance } \\
\hline No & 175 & 24.2 & $* * *$ & 207 & 28.7 & \\
\hline Yes & 78 & 50.3 & & 38 & 24.5 & \\
\hline \multicolumn{7}{|l|}{ Pregnancy complication } \\
\hline No & 149 & 30.2 & & 131 & 26.5 & \\
\hline Yes & 104 & 27.2 & & 114 & 29.8 & \\
\hline \multicolumn{7}{|l|}{ Birth Complication } \\
\hline No & 204 & 28.3 & & 202 & 28 & \\
\hline Yes & 49 & 31.4 & & 43 & 27.6 & \\
\hline \multicolumn{7}{|l|}{ Past health facility delivery } \\
\hline No & 68 & 19.9 & $* * *$ & 83 & 24.3 & \\
\hline Yes & 185 & 34.6 & & 162 & 30.3 & \\
\hline \multicolumn{7}{|l|}{ ANC visits } \\
\hline Less than 4 & 58 & 20.2 & $* * *$ & 85 & 29.6 & \\
\hline
\end{tabular}


Table 6 Bivariate distribution of selected birth companionship variables by demographic and facility characteristics, PQCC Study 2016 (Continued)

\begin{tabular}{|c|c|c|c|c|c|c|}
\hline & \multicolumn{3}{|c|}{ Accompanied by Partner } & \multicolumn{3}{|c|}{ Accompanied by mother-in-law } \\
\hline & No. & $\%$ & $p$-value & No. & $\%$ & $p$-value \\
\hline 4 plus & 192 & 32.8 & & 158 & 27 & \\
\hline \multicolumn{7}{|l|}{ Tribe } \\
\hline Luo & 176 & 30.1 & & 114 & 19.5 & $* * *$ \\
\hline Kuria & 53 & 25.5 & & 114 & 54.8 & \\
\hline Other & 24 & 28.2 & & 17 & 20 & \\
\hline \multicolumn{7}{|l|}{ Religion } \\
\hline Catholic & 72 & 29.8 & & 68 & 28.1 & \\
\hline Protestant/Pentecostal & 46 & 24.1 & & 59 & 30.9 & \\
\hline Seventh Day Adventist & 84 & 31.9 & & 75 & 28.5 & \\
\hline Other Christian & 48 & 28.9 & & 39 & 23.5 & \\
\hline Muslim/other religion & 3 & 20 & & 4 & 26.7 & \\
\hline \multicolumn{7}{|l|}{ Delivery facility type } \\
\hline Gov't Hospital & 120 & 29.7 & & 122 & 30.2 & \\
\hline Gov't HC/Disp & 94 & 26 & & 96 & 26.5 & \\
\hline Mission/Private facility & 39 & 35.1 & & 27 & 24.3 & \\
\hline \multicolumn{7}{|l|}{ Delivery provider } \\
\hline Nurse/Midwife & 190 & 29 & & 188 & 28.7 & \\
\hline Doctor/Clinical Officer & 40 & 29.2 & & 35 & 25.5 & \\
\hline Non-skilled attendant & 6 & 28.6 & & 5 & 23.8 & \\
\hline 1 plus skilled providers & 17 & 27 & & 17 & 27 & \\
\hline \multicolumn{7}{|l|}{ Delivery Provider sex } \\
\hline Male & 100 & 30.4 & & 97 & 29.5 & \\
\hline Female & 142 & 27.6 & & 138 & 26.8 & \\
\hline Both & 11 & 32.4 & & 10 & 29.4 & \\
\hline Total & 253 & 28.8 & & 245 & 27.9 & \\
\hline
\end{tabular}

${ }^{*} p<0.05{ }^{* *} p<0.01{ }^{* * *} p<0.001$

everyone. However, others felt that it was the providers who did not want them to have someone close to them.

"They refused so I just stayed alone in the hospital until I stayed two days in the hospital... It is required that I just stay alone... I felt bad" (MP4)

"They do not always allow somebody to be with you, it is the doctor and you alone. Whoever has brought you is to wait outside" (OP5)

"They do not want someone to be close to you, even after giving birth, they tell them that time for visiting patients is over and they send them away." (CP10)

Some women also thought companions were less likely to be allowed during delivery if they were male compared to if they were female companions.
"I was allowed to be with a woman relative but not a man... The woman relative was allowed for when there is a problem she can assist the nurse, but a male relative they do not allow them to stay." (P10)

\section{Reasons for desiring a companion}

Instrumental support The most common reason for desiring a labor companion was to have someone readily available to attend to their needs. This was important because they often felt helpless in labor, and providers were not with them all the time. Also, because providers were usually busy with other things, the presence of their companion ensured that there was someone readily available to help them if they needed help for things such as going to the bathroom room and to call the provider if they developed a problem or went into the second stage of labor. 
Table 7 Multivariate logistic regression of continuous support during labor and delivery on potential predictors, PQCC study 2016

\begin{tabular}{|c|c|c|c|c|c|c|}
\hline & \multicolumn{6}{|c|}{ Allowed Continuous support during } \\
\hline & \multicolumn{3}{|l|}{ Labor } & \multicolumn{3}{|l|}{ Delivery } \\
\hline & $\mathrm{OR}$ & $95 \% \mathrm{Cl}$ & & $\mathrm{OR}$ & $95 \% \mathrm{Cl}$ & \\
\hline \multicolumn{7}{|l|}{ Age } \\
\hline 15 to 19 years & 1 & {$[1$} & 1] & 1 & {$[1$} & 1] \\
\hline 20 to 29 years & 0.79 & {$[0.46$} & $1.35]$ & 0.86 & {$[0.50$} & 1.47] \\
\hline 30 to 48 years & 0.74 & {$[0.36$} & 1.54] & 0.92 & {$[0.43$} & 1.95] \\
\hline Currently married & 1.35 & {$[0.79$} & 2.29] & 0.98 & {$[0.57$} & 1.69] \\
\hline \multicolumn{7}{|l|}{ Number of births } \\
\hline 1 & 1 & {$[1$} & 1] & 1 & {$[1$} & 1] \\
\hline 2 & 0.61 & {$[0.26$} & 1.43] & 1.24 & {$[0.51$} & 3.04] \\
\hline 3 & $0.37^{*}$ & {$[0.15$} & $0.95]$ & 2.06 & {$[0.77$} & $5.50]$ \\
\hline 4 or more & 0.39 & {$[0.15$} & $1.01]$ & 1.45 & {$[0.53$} & 3.93] \\
\hline \multicolumn{7}{|l|}{ Education } \\
\hline No school/Primary & 1 & {$[1$} & 1] & 1 & {$[1$} & 1] \\
\hline Post-primary/vocational/Secondary & 0.73 & {$[0.47$} & 1.14] & 1.27 & {$[0.83$} & 1.96] \\
\hline College or above & 0.75 & {$[0.36$} & $1.56]$ & 1.21 & {$[0.61$} & 2.39] \\
\hline \multicolumn{7}{|l|}{ Literacy } \\
\hline No, cannot write & 1 & {$[1$} & 1] & 1 & {$[1$} & 1] \\
\hline Yes, but with some difficulty & 1.9 & {$[0.72$} & $5.05]$ & 0.73 & {$[0.22$} & 2.42] \\
\hline Yes, very well & $2.89^{*}$ & {$[1.13$} & 7.37] & 1.92 & {$[0.63$} & 5.90] \\
\hline \multicolumn{7}{|l|}{ Household wealth } \\
\hline Poorest/poorer & 1 & {$[1$} & 1] & 1 & {$[1$} & 1] \\
\hline Middle & $1.73^{*}$ & {$[1.04$} & 2.90] & 0.81 & {$[0.47$} & 1.40] \\
\hline Richer/richest & $1.91^{* *}$ & {$[1.20$} & 3.03] & 1.16 & {$[0.74$} & 1.84] \\
\hline Employed & $1.97^{* *}$ & {$[1.24$} & $3.12]$ & 0.91 & {$[0.58$} & 1.42] \\
\hline Past health facility delivery & $2.19^{*}$ & {$[1.05$} & $4.57]$ & 0.71 & {$[0.32$} & 1.58] \\
\hline 4plus ANC visits & 0.68 & {$[0.46$} & $1.01]$ & 0.82 & {$[0.56$} & 1.20] \\
\hline Birth Complication & 0.67 & {$[0.43$} & $1.04]$ & $0.34^{* * *}$ & {$[0.19$} & $0.60]$ \\
\hline \multicolumn{7}{|l|}{ Tribe } \\
\hline Luo & 1 & {$[1$} & 1] & 1 & {$[1$} & 1] \\
\hline Kuria & $0.57^{*}$ & {$[0.37$} & $0.90]$ & 0.79 & {$[0.48$} & 1.29] \\
\hline Other & 0.79 & {$[0.44$} & 1.43] & 0.79 & {$[0.43$} & 1.45] \\
\hline \multicolumn{7}{|l|}{ Religion } \\
\hline Catholic & 1 & {$[1$} & 1] & 1 & {$[1$} & 1] \\
\hline Protestant/Pentecostal & 0.68 & {$[0.41$} & $1.12]$ & 1.06 & {$[0.62$} & 1.79] \\
\hline Seventh Day Adventist & 0.7 & {$[0.44$} & $1.12]$ & 1.55 & {$[0.99$} & 2.44] \\
\hline Other Christian & 0.79 & {$[0.46$} & $1.35]$ & 0.93 & {$[0.54$} & 1.59] \\
\hline Muslim/other religion & 0.86 & {$[0.21$} & $3.44]$ & 1.8 & {$[0.43$} & 7.52] \\
\hline \multicolumn{7}{|l|}{ Delivery facility type } \\
\hline Gov't Hospital & 1 & {$[1$} & 1] & 1 & {$[1$} & 1] \\
\hline Gov't Health Center & $1.98^{* * *}$ & {$[1.32$} & 2.97] & 1.46 & {$[0.98$} & 2.17] \\
\hline Mission/Private facility & 0.86 & {$[0.49$} & $1.51]$ & 1.67 & {$[0.95$} & 2.95] \\
\hline
\end{tabular}


Table 7 Multivariate logistic regression of continuous support during labor and delivery on potential predictors, PQCC study 2016 (Continued)

\begin{tabular}{|c|c|c|c|c|c|c|}
\hline & \multicolumn{6}{|c|}{ Allowed Continuous support during } \\
\hline & \multicolumn{3}{|l|}{ Labor } & \multicolumn{3}{|l|}{ Delivery } \\
\hline & $\mathrm{OR}$ & $95 \% \mathrm{Cl}$ & & $\mathrm{OR}$ & $95 \% \mathrm{Cl}$ & \\
\hline \multicolumn{7}{|l|}{ Delivery provider } \\
\hline Nurse/Midwife & 1 & {$[1$} & 1] & 1 & {$[1$} & 1] \\
\hline Doctor/Clinical Officer & 0.8 & {$[0.46$} & 1.39] & 1.13 & {$[0.63$} & 2.00] \\
\hline Non-skilled attendant & 1 & {$[0.28$} & $3.60]$ & 0.88 & {$[0.28$} & 2.79] \\
\hline 1 plus skilled providers & $0.27^{* *}$ & {$[0.12$} & $0.63]$ & $0.19^{* *}$ & {$[0.060$} & $0.59]$ \\
\hline \multicolumn{7}{|l|}{ Delivery Provider sex } \\
\hline Male & 1 & {$[1$} & 1] & 1 & {$[1$} & 1] \\
\hline Female & 1.24 & {$[0.82$} & 1.89] & 1.42 & {$[0.92$} & 2.19] \\
\hline Both & $4.68^{* *}$ & {$[1.54$} & 14.2] & $5.40^{* *}$ & {$[1.67$} & 17.5] \\
\hline \multicolumn{7}{|l|}{ Accompanied by } \\
\hline Partner & 0.81 & {$[0.53$} & $1.24]$ & $0.61^{*}$ & {$[0.39$} & $0.97]$ \\
\hline Mother-in-law & 0.92 & {$[0.59$} & 1.44] & 1.19 & {$[0.74$} & 1.91] \\
\hline Mother & 1.3 & {$[0.70$} & 2.42] & 1.01 & {$[0.54$} & 1.90] \\
\hline Sister/Sister in law & $1.85^{*}$ & {$[1.13$} & $3.01]$ & 0.73 & {$[0.45$} & 1.18] \\
\hline Friend- Neighbor & 0.87 & {$[0.51$} & 1.46] & 0.61 & {$[0.35$} & 1.06] \\
\hline Facility crowded & $0.65^{*}$ & {$[0.45$} & $0.93]$ & $1.69^{* *}$ & {$[1.15$} & 2.50] \\
\hline Desired labor companion & $1.42^{*}$ & {$[1.01$} & 2.00] & & & \\
\hline Desired delivery companion & & & & $1.97^{* * *}$ & {$[1.48$} & 2.62] \\
\hline Interviews in community (ref = HF) & 1.02 & {$[0.70$} & 1.49] & 0.79 & {$[0.54$} & 1.14] \\
\hline Postpartum length $=>1$ week $($ ref $<1$ & 0.99 & {$[0.53$} & 1.83] & 1.24 & {$[0.64$} & 2.38] \\
\hline Constant & 0.87 & {$[0.22$} & $3.42]$ & $0.15^{*}$ & {$[0.033$} & $0.64]$ \\
\hline N & 763 & & & 764 & & \\
\hline
\end{tabular}

${ }^{*} p<0.05 * * p<0.01 * * * p<0.001$

"They did not allow them just as the other person has said, you want to be with your relative and they are sent out. You know sometimes they the doctors are out and have gone wherever places. For you your labor is bad and you are bleeding, so the energy that you will have to walk and go to call the doctor you do not have and so it is your relative whom you can send to go and get you the doctor and so that is why I was not happy with this." (WP6)

"I feel it is good to have someone with you because sister [the nurse] can show you the bed to go and sleep, then she goes to sit somewhere else. So if you are about to give birth then you can send her to go and call for you the nurse so that she can come and help you." (CP4)

Companions were also needed to help run errands: like going to buy drugs and supplies that might not be available on the ward or arranging additional help if they were referred.
"I feel that they should be next to you because, the doctor can tell you that there is some drug that you should be treated with, and so he can be sent to go to the chemist to go and buy." (CP1)

"They should be around because sometimes you can develop complications and you are being referred and so if you are alone then you may not be able to get help." (OP6)

In addition, companions were needed after delivery to help care for the baby, including holding the baby when they needed to go to the bathroom.

"They should be allowed. Because they can help you with carrying the baby." (CP10)

"I felt good because there are times you can be going to bath, and so they will remain and take care of the baby for you." (KP5) 
Informational support and advocacy For others, the companion helped meet their informational needs by telling them what to do, helping them make decisions, and advocating for their care-as they felt they were not in a state to make decisions on their own during labor. Others wanted the companion around in order to have someone who could remind them later of what happened during labor.

"They should allow your relatives to be with you at that particular time in case that anything happens then they can share with the nurses to know what next is to be done to you, because at that time, you are in so much pain that you cannot make any wise decision." (KP3)

"For me I like it because at that time I do not know anything that am doing so when she is there then she will be able to tell me that at that time you did this and that until the doctor either did this to you is when you were able to put to birth." (OP3)

Emotional support Emotional support was barely mentioned as a reason for desiring a companion. Only one woman's response to why she was unhappy about being denied a companion related to a desire for emotional support during labor.

"That was not good because when you have someone near, she will try to make your heart relax, yes, but when they refuse you are just alone you will surely have some problems." (MP2)

\section{Reasons for not desiring for a companion}

Some women did not desire companionship during labor, and in particular at the time of delivery, and so did not mind that their companions were not allowed to stay. Reasons for not desiring companionship included the following.

They cannot help you A common reason women gave for not desiring a companion was that "they can't help you." Some mentioned that the reason why they come to deliver in the facility was because their relatives could not help them at home, and so there was no need for them to be around once they had brought them to the facility.

"I do not want because when you left home...they could not help you and so that is why you came to get help from the hospital." (CP4)
Others felt it was only the provider who could help them, and that they felt safe with the providers. Thus, there was no need for their companion to be around.

"I feel that it is the right thing [that companion was asked to wait outside] because whoever has brought you is not the doctor, and he has brought you in safe hands of the doctor and so he should just stay out and wait .... because there is nothing he will do apart from just looking at you and so it is only when the doctor is not able then he will refer you." (OP4)

In particular at the time of delivery, many felt it was the provider who knew everything and so should be the only one with them.

"It is the doctor who knows everything and so he is the one who should be with you alone in the delivery room but the other person I would not like him to be next to me when I am giving birth." (OP3)

Some also felt lay companions, in addition to not being able to help them, might laugh at them if they saw their condition at the time of delivery.

"I should be with the doctor alone because she is the one with all the know-how but this relative of mine, all he will do is just look and laugh at you." (WP2)

Others felt at the time of delivery it was only "between you and your God" and so there was no need for a relative to be there to see their nakedness

"We did not say that it is bad to have someone with you...let them just come. But when time comes that you are in a state of between you and your God, then let them stay outside and wait for you....At that time, he should not see my nakedness." (OP3)

Embarrassment Some women felt embarrassed to have someone other than a provider see them during labor, and particularly during delivery. They also felt that providers had seen a lot to not be surprised at their condition or nakedness, but did not feel the same way about lay companions.

"No, I feel embarrassed and [will] rather be with the doctor alone in that hospital." (CP5)

"I refused because when you are giving birth, maybe you do not have clothes and you are with your mother-in-law so you feel embarrassed." (CP8) 
"It is better if you are with the nurse alone because for her she has seen a lot but the other person can exclaim that 'ei and this person." (CP10)

Certain roles companions were asked to play made women particularly vulnerable, as well as decrease their desire for companionship. For example, one woman reported she felt very embarrassed because the provider asked the relative to hold the flashlight for him to conduct the delivery (because there was no electricity) and she felt completely exposed to this relative.

"I did not like it because whoever escorted me is the one that the doctor made to carry for him the sport light and was pointing it at me and I did not like it at all....but it is only that I was in a problem and I was praying to God to help me, but my inner heart did not like the fact that this person was seeing my nakedness." (OP3)

Fear that support persons may go outside the facility and discuss private matters Some feared that the support person may discuss what they saw about them with others outside the facility. They were confident that providers could not discuss what they saw about them outside the facility, but did not feel the same about lay companions.

"At that time they should not allow someone to be in, the doctor is the only person who should see my nakedness...people are so mouthy. Some people when they leave the hospital they go and say so many strange things about you, how they saw you, but the doctor is not allowed to go out and talk about what has happened in the facility." (KP4)

"For me I don't want because they can look at you then go and tell other women that ei.. so and so is like this." (CP2)

"For me I liked it and felt good [that the person was not allowed inside] because whoever is taking care of you can go out and talk about your secret but the doctor cannot." (CP5)

Fear of abuse Some did not want the companion to stay with them because they feared that they might unknowingly abuse the support person when they are in pain.

"After reaching with her at the door, then he should stay aside because you are very angry at that time and so you can find yourself insulting her." (CP10)
Others feared that the support person may abuse them-like shouting at them at them during labor pains. The fear of being abused was mentioned in relation to the type of support person. Some women felt that their mothers-in-law were more likely to be abusive, hence they preferred to have their mother as a support person.

"it's good to be with a parent when giving birth, like me I was with my mother when I was giving birth and I was attended to well but when you are with your mother in law, when taking care of you, she will shout at you that stop disturbing me but when you are with your mother then she will take good care of you, it's good to be with your mother than any other person." (CP9)

\section{Reasons for not desiring birth companionship from their partner}

Women spontaneously talked about preferring a female relative, and not their partners, as birth companions. Reasons for this included that their partners could not help them and so did not need to be there.

\section{"He will not help you with anything...so it is better for him to be away." (KP2)}

Others did not want to see their partners during the time of delivery because they hated him at that time.

"While I was giving birth, I went with my husband as my mum lives in [another town]. But my husband was waiting for me far away... I really did hate him at that time (...Laughs...) when that time came I felt hatred for him and I told him you sit that side" (CP6)

Another reason was fear that their partners might lose sexual desire for them if they saw them at the time of delivery. It appeared that providers reinforced this belief by using it as a reason to discourage the women from having male partners present at the time of delivery.

"When I am inside, I would want him just to stay outside there... The time the baby is coming out, is not a good sight for a man to be there... They always say that when a woman is delivering and the time the baby is coming out, your body is not looking good so when the husband sees that, after delivery, he will not have a desire (sexual desire) for you. That is what is said and most of us mothers know about this \{laughter...\}. They prevent the husbands from seeing this so that later he may have a desire for you. That is why the nurse will enter inside with you only unless there is something she cannot handle is when he will 
be allowed in (WP4). M: This is what nurses are saying or who?\{All in chorus, nurses...\}"

There were also some cultural beliefs around allowing men in the delivery room. Some mentioned their tribes required a mother-in-law to accompany the women at the time of delivery, because the presence of a man can prolong the delivery. Thus, partners were not allowed even during deliveries conducted by traditional birth attendants [TBA].

My third child I delivered at the TBA place. I was accompanied by my husband and other mother-inlaws. When we arrived there the baby was almost coming out, he was told to stay outside [and] not to go in. This was because the Luo culture says that when your husband goes inside with you where you are going to deliver, he will tie you and the baby might delay in coming out, so it is not good that we go with our husbands to where we are delivering (WP7).

Others did not want their partners present because they wanted them to go home and take care of other children.

"For me in most occasions when am going to deliver, I usually go with my husband, and when we go when we reach, after I have been admitted, Like this one, they asked me whoever he was to me and I told them that he is my husband and they asked me if I wanted him to stay behind and be with me. But I told them that no the children are home alone and so he must go back home as I was in safe hands and he was to go back and only call him in case there is anything that needs his attention then they could call him come back...I did not see the point of having [him] around because when giving birth, even if he is my own husband I do not want him to be with me at that time, I want only a doctor." (KP2)

Providers' perspectives: quantitative and qualitative data from in-depth-interviews with providers

We conducted in-depth-interviews with seven clinical officers, 25 nurses and midwives, and 17 non-clinical staff including cleaners and cooks. Thirty of them worked in public hospitals, 13 in health centers, and 6 in mission hospitals (Table 8). Most of the providers reported that women were usually allowed a companion during labor: $84 \%$ reported that during labor, companions were allowed all or most of the time. Fewer, however, reported that women were usually allowed a companion during delivery: only 39\% reported that during delivery, companions were allowed all or most of the time. Clinical staff were also more likely to report that women were allowed continuous delivery support compared to non-clinical staff. Additionally, compared to female providers, male providers were more likely to report that women were allowed continuous labor and delivery support (Table 8).

\section{Role birth companions play in the facility}

The reasons given by providers for allowing birth companions were generally consistent with those given by women. Providers however also implied that the companions were useful not just to the mother but to them the providers. Providers reported companions sometimes helped them with various chores, such as going to purchase supplies and drugs, calling on them to attend to the women, helping women to and from the delivery bed, holding items like a flashlight during delivery, helping them pick up delivery supplies, and cleaning up. The role of the companion was particularly useful in the face of staff shortages, where only one provider may be on duty.

"You know after that like now we have staff shortage, so maybe after examining a mother maybe I am alone in the facility, I may run to come and see clients here but when they are almost ready, she will come and call me. Even after delivery helping them to do one, two, three, like cleaning the clothes something like that."

"Most of the time we need one caretaker to be around. In case of any eventuality they can be asked to go may be to organize for example in a case of complicated delivery and you want things like gloves somebody at least you can send to get for you the things you want, so most of the times we allow one caretaker to be around the mother who is giving birth."

Some providers insisted that they almost always allow birth companions, because they can't do without them when they are alone assisting with the delivery.

"Rarely do we not allow birth companions, rarely, not unless the mother came alone. There are some who come without Birth companions though during ANC they are encouraged to have with Birth partners, but when they come without they are equally just served as others. But when they come with a birth partner, especially $X$ facility, if they do not assist us I don't know how we would do it, because they do assist us even wheeling the patient to the bedside post delivery they do a lot to us especially to me when I am on duty, they do a lot." 
"We have no problem, if the mother has allowed someone who escorted her to labor to get in then we have no problem with the person as there was a time when a mother was delivering and it is the husband who was holding for us the touch."

Some support staff mentioned that some clinical providers only allowed birth companions who could be helpful to them.

"The delivery room is small so the nurse will only need the person who will assist her get things like cotton wool, bucket for the placenta and water."

"The relative may only come in when it is at night and the lights are off so the care taker gets in to hold for the nurse the torch only."

In addition, although companions were usually not allowed at the time of delivery, they were sometimes called on to help with non-compliant women. This was mostly noted by the non-clinical staff.

"This time is more private, he does not allow anyone to be with the mother unless he is alone and the mother is not cooperative... [that] is when he will call a relative to come and talk to the mother to push the baby."

"No, it is only the nurses who are in there. The person is to wait outside. Only if the woman is difficult to deal with is when they call the caretaker to come in and help them."

\section{Reasons why companions may not be allowed in the ward}

The reasons why birth companions were sometimes not allowed in the ward are related to privacy and ward set up, distrust of companions, lack of confidence in companions in the event of complications, and respecting the woman's wishes.

Privacy and ward set up Most providers reported that companions were sometimes not allowed in the ward because of limited space and overcrowding, hence privacy concerns for other women in the labor and delivery wards. Some also mentioned that the set-up of the labor wards is not conducive for companions, including limited seating, which may cause companions to use beds meant for the laboring woman.

If they are two in labor then now you see the privacy will [be a problem] ...but if that woman is all alone there, then we will just allow, so it is more of the setup which is preventing us but we know... that if they want even their spouses to come to the delivery room they should be allowed but because of the setup and the environment now it is not really conducive.

Privacy was the main reason given for not allowing companions at the time of delivery.

"Yes, we allow them but it is not a normal practice here and at second stage we do not allow them. Here you find maybe they came with them, they stay with them but when we are going to second stage we tell them to wait until the time they have delivered. But that one is done because of space as I was saying. Maybe...the relative of this mother $A$ is there and another mother $B$ is delivering so when I bring these relatives here they will also be watching this mother $B$, so we say let me counsel them when we need them we can always call them after delivery"

Distrust of companions Another set of reasons for sometimes not allowing companions were related to distrust of the companions. Providers had several fears of inappropriate things companions did (or which they thought companions might do), which made them reluctant to allow companions. These included the following:

Fear companions will practice things usually done in home deliveries like giving the laboring woman herbs: Several providers mentioned they sometimes refuse to let the companions into the ward because some of them bring traditional herbs that are thought to hasten labor to the health facility to give to the laboring woman. They also mentioned that some companions attempt to apply fundal pressure and touch women with unclean hands.

"like here they like coming with the herbs, since the nurse is one, you leave the mother with the care taker and by the time you are back, you find that they have place for the mother the herb, so it depends on who has brought the client."
"Sometimes you find the mother in second stage, a mother comes in, there are certain things they usually practice home deliveries, so you can find that they start practicing that so we don't allow that in health care so this is why. Mothers at home give fundal [pressure] and they can touch anywhere with bare hands, yeah so in a normal delivery procedure they remain outside the delivery room."

They were particularly distrustful of older birth companions, particularly mothers-in-law, whom they said 
Table 8 Univariate and Bivariate distribution of Provider data, PQCC study 2016

\begin{tabular}{|c|c|c|c|c|c|c|}
\hline & \multirow{2}{*}{\multicolumn{2}{|c|}{$\begin{array}{l}\text { Characteristics of } \\
\text { Providers }\end{array}$}} & \multicolumn{4}{|c|}{ Percent reporting women are allowed continuous support during } \\
\hline & & & \multicolumn{2}{|c|}{ Labor } & \multicolumn{2}{|c|}{ Delivery } \\
\hline & No. & $\%$ & No. & $\%$ & No. & $\%$ \\
\hline \multicolumn{7}{|l|}{ Facility type } \\
\hline Govt. Hospital & 30 & 61.2 & 22 & 73.3 & 10 & 33.3 \\
\hline Govt. Health Center & 13 & 26.5 & 13 & 100 & 6 & 46.2 \\
\hline Mission Hospital & 6 & 12 & 6 & 100 & 3 & 50 \\
\hline \multicolumn{7}{|l|}{ Position in facility } \\
\hline Clinical officer & 7 & 14.3 & 7 & 100 & 4 & $57.1^{*}$ \\
\hline Nurse/Midwife & 25 & 51 & 19 & 76 & 12 & 48 \\
\hline Support staff & 17 & 34.7 & 15 & 88.2 & 3 & 17.6 \\
\hline \multicolumn{7}{|l|}{ Gender } \\
\hline Male & 14 & 28.6 & 14 & 100 & 9 & $64.3^{*}$ \\
\hline Female & 35 & 71.4 & 27 & 77.1 & 10 & 28.6 \\
\hline \multicolumn{7}{|l|}{ Age } \\
\hline 24 to 39 years & 30 & 61.2 & 26 & 86.7 & 12 & 40 \\
\hline 40 to 58 years & 19 & 38.8 & 15 & 78.9 & 7 & 36.8 \\
\hline \multicolumn{7}{|l|}{ Marital status } \\
\hline Single/Widowed & 8 & 17 & 7 & 87.5 & 3 & 37.5 \\
\hline Married & 39 & 83 & 32 & 82 & 15 & 38.5 \\
\hline \multicolumn{7}{|l|}{ Parity } \\
\hline 0 to 3 & 34 & 72.34 & 28 & 82.4 & 13 & 38.2 \\
\hline 4 to 7 & 13 & 27.66 & 11 & 84.6 & 5 & 38.5 \\
\hline \multicolumn{7}{|l|}{ Feels Facility is crowded } \\
\hline No, never & 14 & 28.6 & 12 & 85.7 & 5 & 35.7 \\
\hline Yes, a few times & 6 & 12.2 & 5 & 83.3 & 3 & 50 \\
\hline Yes, most of the time & 23 & 46.9 & 21 & 91.3 & 9 & 39.1 \\
\hline Yes, all the time & 6 & 12.2 & 3 & 50 & 2 & 33.3 \\
\hline \multicolumn{7}{|c|}{ Women allowed labor support } \\
\hline No, never & 1 & 2 & & & & \\
\hline Yes, a few times & 6 & 12.2 & & & & \\
\hline Yes, most of the time & 12 & 24.5 & & & & \\
\hline Yes, all the time & 29 & 59.2 & & & & \\
\hline \multicolumn{7}{|c|}{ Women allowed delivery support } \\
\hline No, never & 18 & 36.7 & & & & \\
\hline Yes, a few times & 12 & 24.5 & & & & \\
\hline Yes, most of the time & 9 & 18.4 & & & & \\
\hline Yes, all the time & 10 & 20.4 & & & & \\
\hline Total & 49 & 100 & 41 & 83.7 & 19 & 38.8 \\
\hline
\end{tabular}

${ }^{*} p<0.05 * * p<0.01 * * * p<0.001$

were more likely to give women concoctions to try to hasten labor.

"Mostly those old mothers, the grannies, the mother in laws who are old. They believe that once a mother comes to the labor ward to deliver, maybe to get a baby as fast as possible, so once they are around, they can discuss and give the mother some medicine to fasten delivery so [because of] that most of us who are midwives are very keen and we don't want those mothers to come so many of them, they can give herbal medicine or they boil tea concentrated without sugar 
as they believe that this one will hasten the delivery. That act most of us are not ready to entertain."

Fear companions will force mothers to do things against their will: Some providers did not trust companions because they feared they may force women to do things against their will such as forcing the women to take the herbs.

"some mothers come with the herbs and force the laboring woman to drink or chew and so that is why we don't usually allow them to come in where the woman is"

Fear companions may misinform mothers: There was also the suspicion that companions may misinform the laboring women when they are with them in the labor ward, such as asking them to push before they are fully dilated.

"Mmm sometimes you get, okay personally, the mothers who come with a companion, give misinformation to the mothers, they force the mothers to push before they are fully dilated, and that is why many a times I do not allow them because they give a lot of misinformation to the mothers while they are in labor. [It causes] those things like cervical tear which are not supposed to be there."

Fear that companions may misinterpret what they see in the facility and discuss it outside: Providers also sometimes prevented companions from staying in the ward because of fears that when they observe certain procedures and something goes wrong (like the baby dies), the companions will go out and spread rumors that the baby died because of the procedure the provider performed.

"When you have invasive procedure... the companion is not supposed to be around to see, for they can feel that you did that thing wrongly and yet we were helping the mother or the baby. I: Like what procedures? R1: Like vacuum extraction you cannot allow the companion to be there. There is fear they can take the information at home that they did this that killed our baby by using the instruments."

A support staff mentioned that clinical providers also sometimes disallowed companions because of fears that companions will go out and gossip about the woman's condition to others, including adding their own interpretations to what they saw.

Some women after seeing the woman delivering, they go and talk about how the woman's private part is.
Some women also give birth to babies who are white with a lot of whitish things in them, so they go and start gossiping how the woman was having sex until the last minute is why the baby is born dirty. So that is why the nurses want to do everything and clean the baby. [That] is when she is taken to the other room for the relatives and friends to see the baby.

Fear of companions being disrespectful to providers and not taking instructions: Another reason some providers gave for sometimes disallowing companions was that some of them were disrespectful: they talk rudely to them, do not take instructions, want to order the providers around, and are naughty and noisy.

"...some care takers are so stubborn they talk to the nurses as if they know everything that the nurse should be doing."

"Sometimes, some people want to be the nurse and so they give the nurse orders in there. So such people are told to wait in the labor room."

"...also it depends on the understanding as some of them will come and don't want to listen to the instructions of the provider."

Disallowing companions for women with complications Women with chronic medical conditions or pregnancy complications were sometimes not allowed companions because of the following:

Need to keep the woman's health information confidential: Some providers mentioned not allowing companions to maintain the woman's confidentiality, especially if she had a condition like HIV.

"Like when the patient is positive (HIV) the doctor will first talk to her and find out from the patient whom to inform and not everybody because at times is a neighbor who brought her. Also find out from her who will collect the drugs for her. In such a case is only the doctor who will be with the patient until she says whom she would want to be closer to her."

Need to monitor woman frequently: Some also mentioned not allowing companions if the woman had a condition that they needed to monitor frequently.

"Okay, sometimes if a woman has a chronic condition that you need to monitor, so we may not like a lot of relatives to be there but we have to inform the relative 
that we are doing this and this. So you will find at that time [only] the health worker will be so near to the patient to monitor."

Inappropriate companion behavior: Providers were also concerned that in the event of a complication, the companion's behaviors may discourage the woman.

"Sometimes just in case of any complication as those people, the family people, they will tend to change to discourage the patient by the way they behave. Like they are so anxious about the patient and they try to discourage."

"Some relatives are so scared and so they are not allowed to get in because they will make the woman to also be scared."

A provider mentioned fear of companions collapsing because of what they saw, giving them additional work of caring for the companion as well as the woman.

"There are a few cases the condition of a mother does not warrant the relatives to be near the mother because of big number like sometimes we had seen somebody who has escorted the patient after seeing the thing fainted, now you will have double work, you are struggling with the mother and somebody has just fainted. So in such scenarios in some cases we do tell some not to. Or you are seriously resuscitating a child and the person who has escorted the mother is crying, you tell her to be out as we do the resuscitation process."

\section{Respecting the woman and companions wishes}

Sometimes companions were asked to stay outside the labor or delivery ward to respect the woman's wishes.

"If the mother who is in labor says, me I do not want anyone around until I deliver. [1] just preserve that as her right, but most of the times we just allow the relatives to be around."

Other times, it was the companion who did not want to stay with the woman, because some women, in their pain, were cruel to their companions.

R: During labor pains, most of the women become very cruel or very rude. So some of the family members may be afraid to go and be with them in there. [Laughs] We tell her to choose the best person so if she does not want you then we tell you to leave.

\section{Perceptions about partners as birth companions}

Providers spontaneously mentioned that they were more likely to allow continuous labor and delivery support if the companion was a partner. They mentioned that partners are the first selected to support women when there is limited space, because they did not trust other companions.

"Mostly we allow the husband, we allow them to be with the wife but if another care taker, sometimes we don't allow. I: Why, what is the reason? R1: Because you never know who is the caretaker"

"we do not know the caretaker and you know family issues we don't interfere, only the husband we allow in the delivery room if the patient is willing, some patients are not willing; they tell the husband go away"

They, however, acknowledged that many women did not want their partners in the delivery room because they wanted their privacy.

"Maybe the mother will just say let him [partner] or her go outside for sometime. They do not like when they are pushing especially their husbands [to be around]."

"Sometimes it is the husband who has come, and the woman is the one who sends him away so we also tell them to keep off and wait outside as she is putting to birth"

Other times, it was the partners who did not want to be in the delivery room: because they did not want to see the nakedness of women, didn't find it appropriate to enter the room, or because of cultural reasons.

"They are allowed, but men mostly don't come... One said that he didn't want to see the lady naked, he doesn't want to see her being handled during delivery. But us we do allow them"

"I may say its culture, let me say if it's a husband who has brought the wife, some of them do not want to go where the woman is laboring."

Some providers also discouraged partners from being birth companions, due to the perception that the presence of a partner may cause women to desire more attention.

"when the husband is around this might affect her psychologically as when she sees the husband she 
might want the sympathy from him. When the husband is away she will be with the nurses who should take care of her."

\section{Discussion}

This study is the first study, to our knowledge, to comprehensively examine the prevalence and nature of labor and delivery support from the perspective of both mothers and providers in Kenya, and among the first of such studies in SSA. We found that most women are accompanied to the facility for childbirth and most of them want to have a companion stay with them during labor and after delivery, but fewer want that person around at the time of delivery. Women's reasons for desiring a companion were mostly related to having someone around to help them meet their physical needs; more so than for emotional support. Those who expressed that they did not want a companion felt that companions could not help them, were embarrassed to have the companion see their nakedness, feared that companions may gossip about what they saw, or were afraid the companion might abuse them or vice-versa. Most providers acknowledged the importance of having a birth companion but stated that it is often not possible due to privacy concerns and other reasons related to distrust and lack of confidence in companions. Some providers, however, thought of companions' roles as assisting them with non-clinical tasks rather than providing emotional support to women.

The findings from this study support the few other studies in SSA that challenge the assumption that all women want a birth companion [13]. In addition, continuous support is often examined as a single unit, but our findings suggest that combining labor and delivery together may not align with women's preferences. By examining labor and delivery separately we show that women have different desires for companionship at different stages of labor and delivery. Furthermore, we show that having a companion present doesn't guarantee that the woman will be supported or feel supported. Where providers think of companions as providing support to them, women's needs might be overlooked. Nonetheless, women who were allowed CSLD in their last pregnancy were more likely to desire it in the future, suggesting that they valued the experience.

We also extend the discussion on women's preferences for companionship at labor and delivery. Less than a quarter of women mentioned a partner as their preferred companion during labor, and even fewer (only 6\%) wanted a partner as a companion during delivery. Most women preferred a female companion, such as their mother, mother-in-law, sister, sister-in-law, or friend. A few mentioned co-wives, but notably no one mentioned a traditional birth attendant (TBA) as their preferred companion. The reasons for the low preference male partners included fear that their partners might lose sexual desire for them if they saw the baby coming out, as well as cultural beliefs about how the presence of a partner could affect the delivery. The preferred type of companion is also influenced by tribal norms, with a mother-in-law being preferred over the partner among the Kuria tribe. Our findings are consistent with that of a study in Ghana, where $24 \%$ of respondents preferred a male labor companion [13], but much lower than that from a study in Nigeria where most women (86\%) preferred their husband as their labor companion [21]. A reason for this might be that the study in Nigeria involved women with higher socio-economic status than our respondents. We found that wealthier and more educated women were more likely to desire their partners as both labor and delivery companions than poorer and less educated women.

The national guideline for Obstetrics and Perinatal Care in Kenya specifically mentions partners as birth companions, and, currently, there are initiatives to promote male involvement in maternal and child health in Kenya. These may be reasons why providers spontaneously discussed a preference for partners as companions during interviews. The survey results, however, suggest that women are less likely to be allowed continuous support at delivery if the companion was a male partner. In addition, women perceive that providers are less likely to allow them to have continuous labor and delivery support if their companions are male partners. This inconsistency between providers' and women's report might be due to providers responding in a socially desirable manner in spite of the fact that both groups are not particularly supportive of having male partners as birth companions. Moreover, the spontaneous discussion among women about male companions may suggest that they are not entirely supportive of the emphasis on having their partners as companions. These findings suggest that we need to find better ways of changing social norms around the role of men during labor and delivery and promoting the involvement of male partners in maternal and child healthcare, while prioritizing women's preferences.

Morhason-Bello et al., found that emotional support was the most cited reason for women's desire for birth companionship (80\%). This finding is not consistent with that of our study, and the demographics of our sample may explain the difference. The limited reference to emotional support should, however, not be misconstrued as a lack of desire for emotional support. We did not specifically ask our participants about emotional support or other types of support. The reasons women give for desiring labor companions are therefore likely the top 
reasons based on the realities of their situation-which in this case is a need for instrumental support to meet their practical needs at the facility. In addition, Alexander et al., in their study in Ghana, found that male companions were expected to provide emotional support, while female companions were expected to provide other types of support [13]. The limited reference to emotional support in our study may therefore be due to the low preference for male companions.

Beyond women's desires and preferences are health system barriers to providing continuous labor and delivery support. Providers cite overcrowding and privacy issues are the main reasons for disallowing birth companions. This is consistent with our survey results, where women who perceived the facility as crowded were less likely to be allowed a labor companion. It is also consistent with findings related to barriers to promoting CLSD in other low resource settings [8]. Our findings suggest that providers sometimes have to prioritize different aspects of respectful maternity care-and they tend to choose privacy over CSLD. Overcrowding, however, did not predict continuous delivery support in the way we expected-women who felt that the facility was overcrowded were more likely to have continuous delivery support. It is unclear why this is so, but this finding highlights the importance of other factors besides crowding that may affect CSLD.

Providers gave other reasons for disallowing companions, such as mistrust and lack of confidence in companions. In contrast, women frequently expressed confidence and trust in providers, obviating the need for a companion. Providers seemed particularly distrustful of older women, and especially mothers-in-law, who are the most common companions among Kuria women. Kuria women were therefore less likely to be allowed continuous labor support, compared to Luo women, even though they were more likely to have a companion present at the facility. Providers' preference for younger female companions is reflected in women being more likely to be allowed continuous labor support if they had a sister or sister-in-law as their companion. Furthermore, being allowed labor support is predicted by socioeconomic status (SES), with wealthier, employed, and literate women being more likely to be allowed continuous labor support than poorer, unemployed, and illiterate women. This finding is consistent with the literature on differential care in health facilities based SES $[38,39]$. Additionally, women with complications were less likely to be allowed continuous labor and delivery support than those without complications. The reasons for this include, a perceived need by providers to keep women's health information confidential, avoid interruptions during the care process, and protect women from companions who might not be helpful in supporting them to deal with complications. Again, it appears that providers feel the need to prioritize, and companionship is often not considered as a top priority.

Health system weaknesses also dictate the role of companions. The shortage of clinical and support staff and the burden placed on families to purchase medicines and supplies outside of the facility, have caused providers to redefine the role of companions. This is inferred when providers mostly mention instrumental support from companions as a reason for permitting women to have companions. The instrumental role of companions was mentioned as very useful in the face of staff shortages. This perspective may account for companions being more likely to be allowed in health centers, as there are usually fewer providers on duty in lower-level facilities. In addition, providers, just like the women, hardly mentioned emotional support as a reason for companionship. There is likely limited understanding among providers, as well as women, about what emotional support or woman-centered care should look like. These findings imply a complex interaction between women's needs and preferences, as well as providers needs and preferences, within facilities and systems that might not be set up to provide CSLD.

Our findings have some implications for research. First, despite WHO recommendations on birth companionship, there have been very few studies on it in SSA and other low resource settings. We need rigorous studies to better understand the effectiveness of different forms of birth companionship in these settings. For example, when women have a companion present only during labor and not at delivery, does it reduce the positive effects of CSLD and by how much? Also, given most companions in this setting are relatives and not professional support persons like doulas, how can we improve the effectiveness of CSLD? Can we learn from doula programs in developed settings and develop a cadre of providers for CSLD with whom patients can feel comfortable? Can TBAs be trained as doulas and will that be acceptable to women? (noting that none of the women in our sample was accompanied by a TBA and none mentioned a TBA as their preferred companion). Given reasons for not desiring companionship at delivery, should family members be companions during labor and post-partum and another cadre like a doula be companions for delivery? Will doula programs be feasible, acceptable, and sustainable in low resource settings? What level of training for companions is sufficient to have an impact and be cost-effective for low resource settings? Given privacy concerns and larger health systems barriers, what changes can be made to promote labor and delivery support? And how can we address the concerns of both women and providers to ensure that labor and delivery support is effective and reflects their needs and concerns. 
The study has some limitations. First, the data are based on self-report. Social desirability and recall bias are thus potential limitations. Second, the findings may not be generalizable to all women and providers. in Kenya, given the peculiarities of the study County. In addition, the qualitative data may not reflect the perceptions of all women in the study County. Nonetheless, the consistency of these findings with other data from the study suggests some transferability. Despite these limitations, this study makes valuable contributions to the existing literature on social support in low resource settings. It is one of the largest and most comprehensive studies on labor and delivery support in SSA.

\section{Conclusions}

This study provides important insights that can be used to improve practice and policies on birth companionship in low-resource settings. The findings suggest that there are differences in women's desire for companionship at different phases of labor and delivery, as well as their preference for different types of companions-and these are associated with factors such as age, SES, and tribe. Therefore, it is essential for providers to understand and respect women's preferences and desires. For example, women may desire support during labor, delivery, and post-delivery; only during labor or post-delivery; or not at all; these preferences should be respected. It is also essential that providers identify women's preferred companions, and that the person who takes the woman to the facility may not be her preferred companion. Antenatal care is an opportunity to help women understand the importance of emotional support and the need to have their preferred companions with them when they go to the facility to deliver. Training providers, as well as companions, could help address the distrust and lack of confidence in companions as a barrier to allowing CSLD. It will also provide more role clarity and help highlight the value of emotional support during childbirth. In general, we need better definitions of appropriate support and interventions to sensitize women, family, and providers about the role of birth companions. Finally, many health systems in low resource settings do not have the capacity to provide CSLD. Thus, we need structural interventions within health systems to ensure that labor and delivery wards are organized in ways that facilitate CSLD. These include redesigning labor and delivery wards to have adequate space to accommodate companions and partitioning them to ensure patient privacy. Addressing the shortage of health care providers and the lack of needed drugs and supplies within maternity units (which requires family members to acquire them outside the unit) will help reduce the perceived role of the companion as an aide to providers (and one needed to run errands) and increase emphasis on their emotional support role. Without system strengthening it will be difficult to provide truly woman-centered care.

\section{Additional files}

Additional file 1: Interview guides. (DOCX $95 \mathrm{~kb}$ )

Additional file 2: Coding example. (DOCX $96 \mathrm{~kb}$ )

Additional file 3: COREQ checklist. (PDF $66 \mathrm{~kb}$ )

Additional file 4: Multivariate regression of desire for labor companionship and male companions on potential predictors. (DOCX $105 \mathrm{~kb})$

\section{Abbreviations}

CSLD: Continuous support during labor and delivery; FGD: Focus group discussions; PQCC: Perceived Quality of Care during Childbirth; SSA: Sub-Saharan Africa; WHO: World Health Organization

\section{Acknowledgments}

We will like to thank Linet Ouma and Beryl Akinyi Ogola, who organized and moderated the focus group discussions and conducted the provider interviews; and all our survey data collectors. We are grateful to the PTBI-East Africa and PTBI-Kenya teams, PTBI fellowship leaders and PA's other mentors-Craig Cohen, Dominic Montagu, and Audrey Lyndon-for supporting the study. We thank the leadership of Migori County, sub-counties, and health facilities, and the community health extension workers and community health volunteers, who facilitated our entry into the County, facilities, and communities. Finally, we will like to express our gratitude to all our study participants for their willingness to share their birth stories with us.

\section{Funding}

This work was supported by the University of California, San Francisco, Preterm Birth Initiative (PTBi) transdisciplinary post-doctoral fellowship, which is funded by the Bill and Melinda Gates Foundation [OPP1107312]; and Marc and Lynne Benioff. The funders had no role in the study design, data collection and analysis, decision to publish, or preparation of the manuscript.

\section{Availability of data and materials}

The data analyzed for the manuscript are available from the corresponding author on reasonable request.

\section{Authors' contributions}

PA led the design and implementation of the study, conceived the manuscript, conducted the analysis, and led the writing. CK supported the analysis and writing of the manuscript. LK and DW served as mentors to PA and supported in the design and implementation of the study and writing of the manuscript. All authors read and approved the final manuscript.

\section{Ethics approval and consent to participate}

The study was reviewed and approved by the University of California, San Francisco Committee for Human Subjects research (IRB number 15-18458) and the Kenya Medical Research Institute Scientific and Ethics Review Unit (protocol ID KEMRI/SERU/CCR/039/3286). The Migori County health leadership also approved the study to be conducted in the county. All participants provided written informed consent prior to participation, including participants between ages 15 to 18 who were considered emancipated minors and so did not require parental consent.

\section{Competing interests}

The authors declare that they have no competing interests.

\section{Publisher's Note}

Springer Nature remains neutral with regard to jurisdictional claims in published maps and institutional affiliations.

\section{Author details}

'Obstetrics, Gynecology and Reproductive Sciences, UCSF, San Francisco, USA. ${ }^{2}$ Institute for Global Health Sciences, UCSF, San Francisco, USA. ${ }^{3}$ Kenya Medical Research Institute, Nairobi, Kenya. 


\section{Received: 15 November 2017 Accepted: 30 April 2018}

\section{Published online: 10 May 2018}

\section{References}

1. Montagu D, Sudhinaraset M, Diamond-Smith N, Campbell O, Gabrysch S, Freedman $L$, et al. Where women go to deliver: understanding the changing landscape of childbirth in Africa and Asia. Health Policy Plan. 2017;32:1146-52.

2. Graham WJ, Varghese B. Quality, quality, quality: gaps in the continuum of care. Lancet. 2012;379:e5-6.

3. Souza JP, Gülmezoglu AM, Vogel J, Carroli G, Lumbiganon P, Qureshi Z, et al. Moving beyond essential interventions for reduction of maternal mortality (the WHO multicountry survey on maternal and newborn health): a cross-sectional study. Lancet. 2013;381:1747-55.

4. Kinney MV, Boldosser-Boesch A, McCallon B. Quality, equity, and dignity for women and babies. Lancet. 2016; Available from: http://linkinghub.elsevier. com/retrieve/pii/S0140673616315252. Cited 24 Sep 2016.

5. Bohren MA, Hunter EC, Munthe-Kaas HM, Souza JP, Vogel JP, Gülmezoglu AM. Facilitators and barriers to facility-based delivery in low- and middle-income countries: a qualitative evidence synthesis. Reprod Health. 2014;11:71.

6. Tunçalp Ö, Were W, MacLennan C, Oladapo O, Gülmezoglu A, Bahl R, et al. Quality of care for pregnant women and newborns - the WHO vision. BJOG. 2015;122:1045-9.

7. $\mathrm{WHO} \mid$ Standards for improving quality of maternal and newborn care in health facilities. WHO. Available from: http://www.who.int/maternal_child_ adolescent/documents/improving-maternal-newborn-care-quality/en/. Cited 4 Apr 2017.

8. Kabakian-Khasholian T, Portela A. Companion of choice at birth: factors affecting implementation. BMC Pregnancy Childbirth. 2017;17 Available from: http://bmcpregnancychildbirth.biomedcentral.com/articles/10.1186/ s12884-017-1447-9

9. Bohren MA, Hofmeyr GJ, Sakala C, Fukuzawa RK, Cuthbert A. Continuous support for women during childbirth. Cochrane Database Syst Rev. John Wiley \& Sons, Ltd; 2017. Available from: http://onlinelibrary.wiley.com/doi/ 10.1002/14651858.CD003766.pub6/abstract. Cited 29 Jul 2017.

10. Hodnett ED, Gates S, Hofmeyr GJ, Sakala C. Continuous support for women during childbirth. In: The Cochrane Collaboration, Hodnett ED, editors. Cochrane Database Syst. Rev. Chichester, UK: John Wiley \& Sons, Ltd; 2013. Available from: http://doi.wiley.com/10.1002/14651858.CD003766.pub5. Cited 6 Aug 2013.

11. Maimbolwa MC, Sikazwe N, Yamba B, Diwan V, Ransjö-Arvidson A-B. Views on involving a social support person during labor in zambian maternities. J Midwifery Womens Health. 2001;46:226-34.

12. Banda G, Kafulafula G, Nyirenda E, Taulo F, Kalilani L. Acceptability and experience of supportive companionship during childbirth in Malawi. BJOG. 2010;117:937-45.

13. Alexander A, Mustafa A, Emil SAV, Amekah E, Engmann C, Adanu R, et al. Social Support During Delivery in Rural Central Ghana: A Mixed Methods Study of Women's Preferences for and Against Inclusion of a Lay Companion in the Delivery Room. J Biosoc Sci. 2014;46:669-85.

14. Berkman LF, Glass T, Brissette I, Seeman TE. From social integration to health: Durkheim in the new millennium. Soc Sci Med. 2000;51:843-57.

15. Rosen P. Supporting women in labor: analysis of different types of caregivers. J Midwifery Women's Health. 2004;49:24-31.

16. Srivastava A, Avan Bl, Rajbangshi P, Bhattacharyya S. Determinants of women's satisfaction with maternal health care: a review of literature from developing countries. BMC Pregnancy Childbirth. 2015;15:97.

17. Diamond-Smith N, Sudhinaraset M, Melo J, Murthy N. The relationship between women's experiences of mistreatment at facilities during childbirth, types of support received and person providing the support in Lucknow, India. Midwifery. 2016;40:114-23.

18. Abuya T, Warren CE, Miller N, Njuki R, Ndwiga C, Maranga A, et al. Exploring the prevalence of disrespect and abuse during childbirth in Kenya. PLoS One. 2015;10. Available from: http://www.ncbi.nlm.nih.gov/pmc/articles/ PMC4401776/. Cited 31 Aug 2015.

19. Ministry of Public Health and Sanitation and Ministry of Medical Services. National Guidelines for quality obstetrics and perinatal care, Kenya. 2012. Available from: https://www.k4health.org/toolkits/kenya-health/nationalguidelines-quality-obstetrics-and-perinatal-care. Cited 26 Aug 2017.
20. Kabakian-Khasholian T, El-Nemer A, Bashour H. Perceptions about labor companionship at public teaching hospitals in three Arab countries. Int J Gynaecol Obstet. 2015;129:223-6.

21. Morhason-Bello IO, Olayemi O, Ojengbede OA, Adedokun BO, Okuyemi OO, Orji B. Attitude and preferences of Nigerian antenatal women to social support during labour. J Biosoc Sci. 2008;40:553-62.

22. Burke Johnson R, Onwuegbuzie AJ, Turner LA. Toward a definition of mixed methods research. J Mix Methods Res. 2007;1:112-33.

23. Tariq S, Woodman J. Using mixed methods in health research. JRSM Short Rep. 2013;4. Available from: https://www.ncbi.n/m.nih.gov/pmc/articles/ PMC3697857/. Cited 5 Jan 2018

24. Migori County Health at a Glance. Available from: https://www. healthpolicyproject.com/pubs/291/Migori\%20County-FINAL.pdf. Cited 2 Jun 2017.

25. Kenya National Bureau of Statistics, Ministry of Health, National AIDS Control Council, Kenya Medical Research Institute, National Council for Population and Development, Nairobi, Kenya, and The DHS Program, ICF International, Rockville, Maryland, USA. The DHS Program - Kenya: DHS, 2014 - Final Report (English). 2015. Available from: http://dhsprogram.com/publications/ publication-FR308-DHS-Final-Reports.cfm. Cited 18 Feb 2016.

26. Migori County fact sheet, 2017. Available from: https://www.afidep.org/ download/fact-sheets/Migori-County-fact-sheetF.pdf. Cited 12 Sep 2017.

27. Health Policy Project. Kenya County Health Fact Sheets. 2015. Available from: https://www.healthpolicyproject.com/index.cfm?id=kenyaCHFS. Cited 2 Jun 2017.

28. Thaddeus S, Maine D. Too far to walk: maternal mortality in context. Soc Sci Med. 1994;38:1091-110.

29. Gabrysch S, Campbell OM. Still too far to walk: literature review of the determinants of delivery service use. BMC Pregnancy Childbirth. 2009;9:34.

30. Afulani PA, Moyer C. Explaining disparities in use of skilled birth attendants in developing countries: a conceptual framework. PLoS One. 2016;11: e0154110.

31. Bohren MA, Vogel JP, Hunter EC, Lutsiv O, Makh SK, Souza JP, et al. The mistreatment of women during childbirth in health facilities globally: a mixed-methods systematic review. PLoS Med. 2015;12:e1001847.

32. Karanja S, Gichuki R, Igunza P, Muhula S, Ofware P, Lesiamon J, et al. Factors influencing deliveries at health facilities in a rural Maasai Community in Magadi sub-county, Kenya. BMC Pregnancy Childbirth. 2018;18:5.

33. Wanjira C, Mwangi M, Mathenge E, Mbugua G, Ng'ang'a Z. Delivery practices and associated factors among mothers seeking child welfare services in selected health facilities in Nyandarua south district, Kenya. BMC Public Health. 2011;11:360.

34. Afulani PA, Diamond-Smith N, Golub G, Sudhinaraset M. Development of a tool to measure person-centered maternity care in developing settings: validation in a rural and urban Kenyan population. Reprod Health. 2017:14:118.

35. Harris PA, Taylor R, Thielke R, Payne J, Gonzalez N, Conde JG. Research electronic data capture (REDCap) - a metadata-driven methodology and workflow process for providing translational research informatics support. J Biomed Inform. 2009:42:377-81.

36. Afulani PA, Kirumbi L, Lyndon A. What makes or mars the facility-based childbirth experience: thematic analysis of women's childbirth experiences in western Kenya. Reprod Health. 2017;14. Available from: https://www.ncbi. nlm.nih.gov/pmc/articles/PMC5747138/. Cited 7 Jan 2018.

37. Braun V, Clarke V. Using thematic analysis in psychology. Qual Res Psychol. 2006;3:77-101.

38. Andersen HM. "Villagers": differential treatment in a Ghanaian hospital. Soc Sci Med. 2004;59:2003-12.

39. Afulani PA. Rural/Urban and socioeconomic differentials in quality of antenatal care in Ghana. PLoS One. 2015;10:e0117996. 